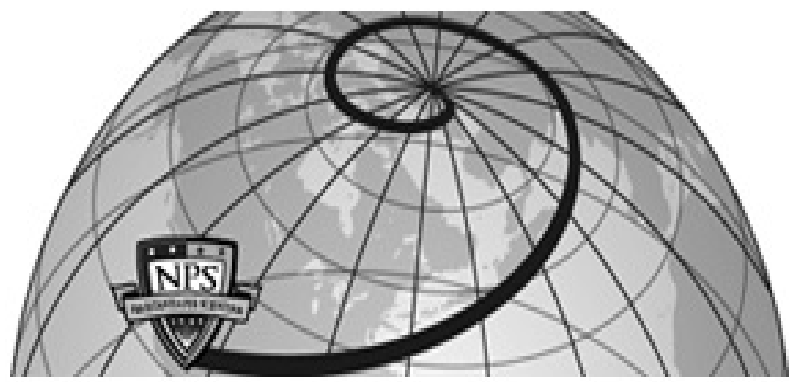

Calhoun: The NPS Institutional Archive DSpace Repository

\title{
Buoyancy Effects of a Growing, Isolated Dendrite
}

Canright, David R.; Davis, S. H.

Journal of Crystal Growth / Volume 114, Issue 41276, 153-185

https://hdl.handle.net/10945/25505

This publication is a work of the U.S. Government as defined in Title 17, United States Code, Section 101. Copyright protection is not available for this work in the United States.

Downloaded from NPS Archive: Calhoun

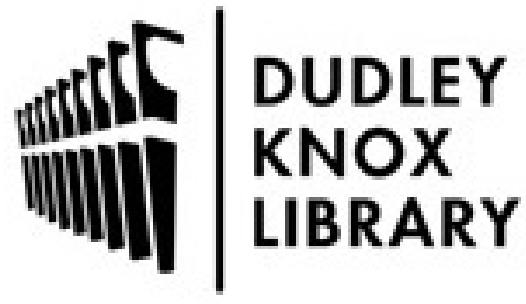

http://www.nps.edu/library
Calhoun is the Naval Postgraduate School's public access digital repository for research materials and institutional publications created by the NPS community. Calhoun is named for Professor of Mathematics Guy K. Calhoun, NPS's first appointed -- and published -- scholarly author.

Dudley Knox Library / Naval Postgraduate School 411 Dyer Road / 1 University Circle Monterey, California USA 93943 


\title{
BUOYANCY EFFECTS OF A GROWING, ISOLATED DENDRITE
}

\author{
D. Canright \\ Mathematics Dept., Code MA/Ca \\ Naval Postgraduate School \\ Monterey, CA 93943
}

\author{
S. H. Davis \\ Engineering Sciences \& Applied Mathematics \\ The McCormick School of Engineering and Applied Science \\ Northwestern University \\ Evanston, IL 60208
}

\begin{abstract}
An axisymmetric dendrite of pure material solidifies downward into an undercooled melt. Surface energy and kinetic undercooling are negligible. The Ivantsov [1] parabolic dendrite is modified by buoyant convection. We construct an approximate solution to the growth/convection problem in powers of a buoyancy parameter $G$. The solution depends on Prandtl number $P$ and Stefan number $S$ (undercooling). When $P$ and/or $S$ are large enough, buoyancy enhances growth and distorts the dendrite by sharpening the tip and widening the base. These results compare well with the experiments on succinonitrile $(P=23)$ of Huang and Glicksman [2] and the local theory of Ananth and Gill [3] up to $G \approx 1000$, but overpredict convective effects for larger $G$. When $P$ and $S$ are small enough, buoyancy slows growth and flattens the tip. Physical explanations are given for the differences in buoyant effects at different $P$. The results suggest that near-tip effects of buoyancy should be different in metallics than in organics.
\end{abstract}




\section{INTRODUCTION}

Solidification processes are important in a variety of contexts, and are of particular interest in the processing of materials. The small-scale details of the solidification, including convection in the melt, can greatly affect the microstructure of the solidified material, and hence the bulk properties of the final product [4]. In many typical situations, the interface between the solid and liquid becomes morphologically unstable as it grows; small bumps grow into long fingers, which in turn grow side branches, yielding tree-like stuctures called dendrites. Experiments have shown that the growing tips of the dendrites are often closely approximated by axisymmetric paraboloids for many materials (or elliptic paraboloids for some others) [5].

The present work considers the near-tip regions of dendrites growing into undercooled liquid and the effects on them of buoyant flow of the nearby fluid warmed by the release of latent heat. There has been much previous work on dendrites with convection, although a relatively small proportion applies directly to natural convection near isolated dendrites. The experimental study by Huang and Glicksman [2,6] on succinonitrile shows that, when the growth is relatively slow, buoyant convection can significantly affect both the growth speeds and the tip radii of isolated dendrites. They found that dendrites growing downward grew faster, with smaller tips, than those growing upward under the same conditions. Similar experiments by Tirmizi and Gill [7], for an ice-water system, shows qualitatively similar results with platelike dendrites, and with the directions down and up switched, due to the fact that water increases in density with temperature near the freezing point.

Ananth and Gill [3] present a theoretical analysis of a single dendrite growing vertically downward into a pure undercooled melt, neglecting surface energy and attachment kinetics at the interface. They assume that the dendrite is a paraboloid, and apply a coordinate expansion, formally valid within a fraction of a tip radius $R$ from the tip. They include the nonlinear effects of buoyancy and derive a pair of coupled nonlinear ordinary differential equations for the temperature and stream function in this region. They solve these equations numerically for various choices of the parameters, the Peclet number and the Grashof number (based on $R$ ), for one particular value of the Prandtl number (23.1), and determine the Stefan number (undercooling). To compare the results with the experiments of Huang and Glicksman [2], for each Stefan number they choose a Grashof number in order to match the experimental Peclet number. From this, they determine the tip radius $R$ and the growth speed $V$; these results compare well with the experiments over the whole range of the data.

In the present work we reconsider the problem treated by Ananth and Gill, in which a dendrite with neither surface tension nor kinetic undercooling grows downward. Rather than use a coordinate expansion, we use a perturbation series in a buoyancy parameter $G$ (the product of the Grashof number, based on the thermal boundary layer thickness, and the square of the Prandtl number). This approach gives a different range of applicability than that of Ananth and Gill; while their method applies for arbitrarily large buoyancy $G$ but only very near the dendrite tip, our approach applies throughout a much larger region about the tip but only when buoyancy is small. Further, rather than presuming a paraboloidal tip, perturbations to the shape of the dendrite are allowed and determined as part of the solution. 
We derive an analytic solution to first order in $G$, with explicit dependence on the Prandtl number $P$ and the Stefan number $S$, for the local velocity and thermal fields and also the buoyant alteration of the interface shape. (The same solution applies to the analogous isothermal problem for a binary alloy, with buoyancy created from concentration variations.) Due to buoyant convection, far from the tip the dendrite is found to widen more quickly than a paraboloid. When the Prandtl number $P$ is moderate to large, buoyant flow is found to enhance growth at the dendrite tip as measured by the Peclet number. Applying a tip-stability criterion [2] allows the selection of a unique growth speed and tip radius for a given Peclet number; this then shows that buoyancy both increases the growth speed and decreases the tip radius. These results agree with the experiments of Huang and Glicksman on succinonitrile in the range where buoyancy is relatively small (up to $G \approx 1000$ ), and for higher $G$ they agree qualitatively but overestimate convective effects. However, when $P$ is small, there is a range of undercooling $S$ for which buoyant convection diminishes growth at the tip, giving a lower Peclet number, and, using the same selection criterion, results in slower growth with a larger tip. These results suggest that buoyancy effects for metals (low $P$ ) may be qualitatively different from those for organics (high $P$ ).

\section{FORMULATION}

Consider a smooth dendrite growing into pure, undercooled, quiescent melt. The dendrite is axisymmetric, and it grows downward along the gravity vector (see Figure 1); the growth is steady in the reference frame of the solid-liquid interface. Surface energy is ignored and thermodynamic equilibrium holds on the interface so that the liquid solidifies at the equilibrium melting temperature $T_{m}$. As a result, the entire solid phase is isothermal, at $T_{m}$. The material properties in each phase are assumed constant, with no density change upon solidification, and the Boussinesq approximation is applied in the melt. Then, the governing equations in the melt are the conservation of energy, mass, and momentum:

$$
\begin{aligned}
\mathbf{u} \cdot \nabla T & =\kappa \nabla^{2} T \\
\nabla \cdot \mathbf{u} & =0 \\
(\mathbf{u} \cdot \nabla) \mathbf{u} & =-\frac{1}{\rho} \nabla p+\nu \nabla^{2} \mathbf{u}+\alpha\left(T-T_{\infty}\right) g \hat{\mathbf{z}},
\end{aligned}
$$

with the boundary conditions

$$
\begin{aligned}
\text { at the interface: } \quad T & =T_{m}, \\
\mathbf{u} & =V \hat{\mathbf{z}}, \\
\rho L V \hat{\mathbf{z}} \cdot \hat{\mathbf{n}} & =k(\nabla T) \cdot \hat{\mathbf{n}}, \\
T & \rightarrow T_{\infty}, \\
\mathbf{u} & \rightarrow V \hat{\mathbf{z}} .
\end{aligned}
$$

Here $T$ is the temperature, $T_{\infty}$ is the temperature of the undisturbed melt, $\kappa$ is the thermal diffusivity, $k$ is the thermal conductivity, $\alpha$ is the thermal expansion coefficient, $L$ is the latent heat (per unit mass), $\mathbf{u}$ is the velocity (relative to the interface), $V$ is the axial 
growth speed of the dendrite, $\rho$ is the density, $p$ is the reduced pressure, $\nu$ is the kinematic viscosity, $g$ is the acceleration of gravity, $\hat{\mathbf{z}}$ is a unit vector upward, and $\hat{\mathbf{n}}$ is a unit vector normal to the interface pointing out of the solid. Condition (2.6) balances the latent heat released at the interface with the heat removed by conduction into the melt, while (2.5) and (2.8) say that the solid and the undisturbed melt are motionless in the laboratory reference frame.

To nondimensionalize the problem, a length or velocity scale is required. Near the dendrite tip, the relevant length scales are the tip radius $R$, the thermal boundary layer thickness $\kappa / V$, and the viscous boundary layer thickness $\nu / V$. All of these involve one of the unknowns, either tip radius $R$ or growth speed $V$. As a result, the dimensionless solution predicts the growth Peclet number $P e=R V / 2 \kappa$, but cannot predict $R$ and $V$ separately. This problem of selecting which of a family of solutions is realized in nature is inherent to the zero-surface-energy problem posed, and is common to the orginal (nonbuoyant) solution of Ivantsov [1], the analysis of Ananth and Gill [3], and the present work. For the determination of a unique physical solution, an additional selection criterion is needed, e.g., one based on the morphological stability of the tip of a dendrite with surface energy. Several such criteria, including those due to Mullins and Sekerka [8], Oldfield [9], Langer and Muller-Krumbhaar [10], and Doherty et. al. [11], are reviewed in Ref. [2].

We nondimensionalize the temperature as $\tilde{T}=\left(T-T_{\infty}\right) /\left(T_{m}-T_{\infty}\right)$, the velocity by $\tilde{\mathbf{u}}=\mathbf{u} / V$, and lengths by the thermal boundary layer thickness $\tilde{\mathbf{x}}=\mathbf{x} /(\kappa / V)$, then for convenience drop the tildes. We introduce an axisymmetric stream function $\Psi$ and use a vorticity formulation to eliminate the pressure. Then the dimensionless equations for energy, stream function, and vorticity are as follows:

$$
\begin{aligned}
\mathbf{u} \cdot \nabla T & =\nabla^{2} T, \\
\omega & =-\nabla^{2}\left(\frac{\Psi}{r} \hat{\boldsymbol{\phi}}\right), \\
(\mathbf{u} \cdot \nabla) \omega-(\boldsymbol{\omega} \cdot \nabla) \mathbf{u} & =P \nabla^{2} \boldsymbol{\omega}-G \frac{\partial T}{\partial r} \hat{\boldsymbol{\phi}},
\end{aligned}
$$

with the boundary conditions

$$
\begin{aligned}
\text { at the interface: } \quad & =1, \\
\Psi & =r^{2} / 2, \\
\nabla \Psi & =r \hat{\mathbf{r}}, \\
\hat{\mathbf{z}} \cdot \hat{\mathbf{n}} & =S(\nabla T) \cdot \hat{\mathbf{n}}, \\
T & \rightarrow 0, \\
\Psi & \rightarrow r^{2} / 2,
\end{aligned}
$$

where

$$
\mathbf{u}=\nabla \times\left(\frac{\Psi}{r} \hat{\phi}\right) \quad, \quad \omega=\omega \hat{\phi}=\nabla \times \mathbf{u}
$$

Here $(r, \phi, z)$ are cylindrical coordinates with corresponding unit vectors $(\hat{\mathbf{r}}, \hat{\boldsymbol{\phi}}, \hat{\mathbf{z}}), \boldsymbol{\omega}$ is the vorticity vector with the azimuthal component $\omega$, and the three dimensionless parameters 
are the Stefan number (undercooling) $S \equiv\left(T_{m}-T_{\infty}\right) c_{p} / L$ with $c_{p}$ the specific heat, the Prandtl number $P \equiv \nu / \kappa$, and the gravitational parameter $G \equiv g \alpha\left(T_{m}-T_{\infty}\right) \kappa / V^{3}$. $(G$ is the product of the Grashof number based on the thermal boundary layer thickness and the square of the Prandtl number.) The undercooling $S$ must be smaller than unity for the rate of solidification to be limited by thermal diffusion, as assumed here; otherwise, the solidification is very rapid and some other effect, such as attachment kinetics, must limit growth [12].

The problem formulated above, of solidification of a pure substance into an undercooled melt, limited by diffusion of released latent heat, is analogous to a solidification problem for a binary alloy, under certain assumptions [13]. For a two-component mixture, the solid and liquid phases in local thermodynamic equilibrium at the interface generally have different compositions. For example, considering the major component the solvent and the other the solute, typically the solute is less soluble in the solid phase, i.e., its concentration is smaller in the solid than in the liquid. Thus, as solidification proceeds, not only is latent heat released, but also the excess solute. When the mass diffusivity $D$ is much smaller than the thermal diffusivity $\kappa$ (the usual case) and the latent heat $L$ is not large, then the speed of solidification is limited by diffusion of the rejected solute into the melt, and the process is nearly isothermal. Formally, if latent heat and thermal variations are negligible, then this alloy problem reduces to the identical dimensionless form above, with the following correspondences: $T \rightarrow C, \kappa \rightarrow D, S \rightarrow\left(C_{l}-C_{\infty}\right) /\left(C_{l}-C_{s}\right)$, $P \rightarrow S c \equiv \nu / D, G \rightarrow g \beta\left(C_{l}-C_{\infty}\right) D / V^{3}$, where $C$ is concentration of solute, with values $C_{s}$ in the solid, $C_{l}$ in the liquid at the interface, and $C_{\infty}$ deep into the melt, $D$ is the diffusivity of the solute in the melt, $S c$ is the Schmidt number, and $\beta$ is a solutal coefficient of expansion, defined by $\left(\rho-\rho_{\infty}\right) / \rho_{\infty}=\beta\left(C-C_{\infty}\right)$. Therefore, the perturbation solution derived below applies to this binary solidification problem controlled by mass diffusion, as well as the case of a pure substance whose growth is limited by thermal diffusion.

\section{PERTURBATION SOLUTION}

The problem (2.9-2.18) presents several difficulties. The interface between the solid and liquid phases is a free boundary, and the energy flux condition at the interface (2.15) is nonlinear, as are the convection terms in the transport equations (2.9) and (2.10). However, when buoyancy is absent $(G=0)$, a solution is well known, due to Ivantsov [1]. In this case, there is no motion of material (in the reference frame of the quiescent melt), and the solid-liquid interface is a paraboloid of revolution, moving steadily along its axis as the liquid solidifies. In the reference frame of the interface, the flow is uniform everywhere $(\mathbf{u}=\hat{\mathbf{z}})$.

To get some indication of the effects of gravity while avoiding the difficulties of the nonlinear convection terms, we consider the case where buoyancy is relatively small $(G \rightarrow$ 0 ), and seek a solution as a perturbation to the Ivantsov solution. We write

$$
\begin{aligned}
& T=T_{0}+G T_{1}+\cdots, \\
& \Psi=\Psi_{0}+G \Psi_{1}+\cdots, \\
& \omega=\omega_{0}+G \omega_{1}+\cdots,
\end{aligned}
$$

At leading order we have the Ivantsov solution, which is most easily expressed in 
axisymmetric paraboloidal coordinates $(\xi, \eta, \phi)$ as shown in Figure 2, where

$$
\xi=\frac{\sqrt{r^{2}+z^{2}}+z}{2} \quad, \quad \eta=\frac{\sqrt{r^{2}+z^{2}}-z}{2},
$$

so that $r=2 \sqrt{\xi \eta}, \quad z=\xi-\eta$,

and $\phi$ is the azimuthal angle. (Note that these are not the same parabolic coordinates used by Ananth and Gill [3].) The thermal field $T_{0}$ and the translational velocity are as follows:

$$
T_{0}=\frac{E_{1}(\eta)}{E_{1}\left(H_{0}\right)} \quad, \quad \Psi_{0}=\frac{r^{2}}{2}=2 \xi \eta \quad, \quad \omega_{0}=0
$$

and the position of the interface is given by the paraboloid $\eta=H_{0}$, where the constant $H_{0}$ satisfies

$$
H_{0} e^{H_{0}} E_{1}\left(H_{0}\right)=S
$$

The (dimensionless) radius of curvature at the tip of this paraboloid is $2 H_{0}$, so at this order the Peclet number is $P e=R V / 2 \kappa=H_{0}+\mathrm{O}(G)$. Here $E_{1}$ is the exponential integral given by:

$$
E_{n}(x) \equiv \int_{1}^{\infty} \frac{e^{-x s}}{s^{n}} d s
$$

following Abramowitz and Stegun [14].

To lowest order the interface is a paraboloid whose dimensionless size is determined by the undercooling $S$. We explicitly consider buoyant effects on the shape of the dendrite by expanding the interface position also in a regular perturbation in $G$ :

$$
\eta=H(\xi)=H_{0}+G H_{1}(\xi)+\cdots \quad,
$$

At order $G$ we first see the effects of buoyancy. The equations are:

$$
\begin{aligned}
\xi \frac{\partial T_{1}}{\partial \xi}-\eta \frac{\partial T_{1}}{\partial \eta}-\frac{1}{2} \frac{\partial \Psi_{1}}{\partial \xi} T_{0}^{\prime} & =\frac{\partial}{\partial \xi}\left(\xi \frac{\partial T_{1}}{\partial \xi}\right)+\frac{\partial}{\partial \eta}\left(\eta \frac{\partial T_{1}}{\partial \eta}\right) \\
\omega_{1} & =\frac{-1}{2(\xi+\eta) \sqrt{\xi \eta}}\left[\xi \frac{\partial^{2} \Psi_{1}}{\partial \xi^{2}}+\eta \frac{\partial^{2} \Psi_{1}}{\partial \eta^{2}}\right], \\
\xi \frac{\partial \omega_{1}}{\partial \xi}-\eta \frac{\partial \omega_{1}}{\partial \eta} & =P\left[\sqrt{\xi} \frac{\partial^{2}}{\partial \xi^{2}}\left(\sqrt{\xi} \omega_{1}\right)+\sqrt{\eta} \frac{\partial^{2}}{\partial \eta^{2}}\left(\sqrt{\eta} \omega_{1}\right)\right]-\sqrt{\xi \eta} T_{0}^{\prime}
\end{aligned}
$$

with the boundary conditions

$$
\begin{array}{ll}
\text { at } \eta=H_{0}: \quad & T_{1}+H_{1} T_{0}^{\prime}=0, \\
& \Psi_{1}=\frac{\partial \Psi_{1}}{\partial \eta}=0, \\
& H_{1}+\xi H_{1}^{\prime}=-S\left[H_{1} T_{0}^{\prime}+H_{1} H_{0} T_{0}^{\prime \prime}+H_{0} \frac{\partial T_{1}}{\partial \eta}\right], \\
\text { as } \eta \rightarrow \infty: \quad \Psi_{1} \rightarrow 0, \quad \Psi_{1} \rightarrow 0,
\end{array}
$$


where primes denote derivatives.

The flow at this order is driven only by the lowest order temperature gradient. So we can solve for the perturbation flow first, from (3.8), (3.9), (3.11), and (3.13b), and then use the flow to find the temperature and interface shape perturbations.

Because the driving temperature gradient $T_{0}^{\prime}$ depends only on coordinate $\eta$ and the interface conditions are prescribed on the coordinate surface $\eta=H_{0}$, we seek a solution that is separable in parabolic coordinates. If we assume that the vorticity is of the form

$$
\omega_{1}(\xi, \eta)=\sqrt{\frac{\xi}{\eta}} f(\eta)
$$

then the vorticity equation (3.9) becomes

$$
P f^{\prime \prime}+f^{\prime}-\frac{f}{\eta}=T_{0}^{\prime}=\frac{-e^{-\eta}}{\eta E_{1}\left(H_{0}\right)}
$$

The general solution is

$$
\omega_{1}=\frac{-1}{(P-1) E_{1}\left(H_{0}\right)} \sqrt{\frac{\xi}{\eta}}\left[E_{2}(\eta)+A E_{2}\left(\frac{\eta}{P}\right)+B \eta\right],
$$

where $A$ and $B$ are arbitrary constants.

The corresponding stream function is not in simple separable form, due to the combination $(\xi+\eta)$ in the denominator of the coefficient in (3.8). However, we can assume a stream function of the form

$$
\Psi_{1}(\xi, \eta)=\xi^{2} g(\eta)+\xi h(\eta)
$$

and find that (3.8) becomes

$$
\begin{aligned}
& \eta g^{\prime \prime}=-2 f \\
& \eta h^{\prime \prime}=-2(\eta f+g)
\end{aligned}
$$

Thus, the general solution for the stream function is

$$
\begin{aligned}
\Psi_{1}=\frac{2}{(P-1) E_{1}\left(H_{0}\right)}\left\{\xi^{2}\left[g_{1}(\eta)+A P g_{1}\left(\frac{\eta}{P}\right)+B \frac{\eta^{2}}{2}+C \eta+D\right]\right. \\
\left.+\xi\left[h_{1}(\eta)+A P^{2} h_{1}\left(\frac{\eta}{P}\right)-C \eta^{2}+2 D \eta(1-\log \eta)+E \eta+F\right]\right\},
\end{aligned}
$$

where $g_{1}(\eta) \equiv E_{2}(\eta)-E_{3}(\eta), h_{1}(\eta) \equiv 2 E_{3}(\eta)-E_{2}(\eta)$, and $C, D, E$, and $F$ are arbitrary constants; $A$ and $B$ are the constants inherited from $\omega_{1}$.

The boundary conditions on the separable parts $g(\eta)$ and $h(\eta)$ (i.e., the two functions in square brackets above) are

$$
\begin{array}{ll}
\text { at } \eta=H_{0}: & g=g^{\prime}=0 \quad, \quad h=h^{\prime}=0 \quad, \\
\text { as } \eta \rightarrow \infty: & g \rightarrow 0 \quad, \quad h \rightarrow 0 .
\end{array}
$$


We can find a locally valid solution of (3.20) that satisfies the interface conditions (3.21), but not the conditions at infinity (3.22). This solution is valid within a distance $O\left(G^{-1}\right)$ from the tip in all directions; we call this the tip solution. (See Figure 3.) Along the dendrite, far from the tip, the nonlinear effects of buoyant convection become important. We expect a strongly convecting boundary-layer in this region, but as it is far downstream from the tip, it should have negligible effects in the tip region. Far from the dendrite, a distance of $O\left(G^{-1}\right)$, is well outside the thermal and viscous boundary layers (unless $\left.P \sim G^{-1}\right)$. In this outer region, the fluid is isothermal $(T=0)$ and the flow is irrotational; it matches the inner flow to the uniform flow of the undisturbed fluid. That is, far from the dendrite, the (potential) flow departs from separable form to conserve mass. So we seek a local solution about the tip that becomes potential flow far away.

We choose $B=0$ so the vorticity decays in $\eta$. The corresponding terms represent Pouiselle flow "without the pipe," $\mathbf{u}=r^{2} \hat{\mathbf{z}}$. We also choose $C=0$ because the corresponding terms, which represent stagnation point flow $(\mathbf{u}=-r \hat{\mathbf{r}}+2 z \hat{\mathbf{z}})$, grow faster in $\eta$ than the remaining terms. Another reason to discard the flow terms with coefficients $B$ and $C$ is that they would imply that the dendrite induces strong flow far ahead of the tip, which is physically unreasonable.

Then the other constants are given by the interface conditions (3.21):

$$
\begin{aligned}
A & =\frac{-g_{1}^{\prime}\left(H_{0}\right)}{g_{1}^{\prime}\left(H_{0} / P\right)}, \\
D & =-\left[g_{1}\left(H_{0}\right)+A P g_{1}\left(H_{0} / P\right)\right], \\
E & =-\left[h_{1}^{\prime}\left(H_{0}\right)+A P h_{1}^{\prime}\left(H_{0} / P\right)-2 D \log H_{0}\right], \\
F & =-\left[h_{1}\left(H_{0}\right)+A P^{2} h_{1}\left(H_{0} / P\right)+2 D H_{0}\left(1-\log H_{0}\right)+E H_{0}\right]=0
\end{aligned}
$$

(It can be shown that $F$ is identically zero.) This completes the tip flow solution.

To interpret these results, note that the flow terms $g_{1}(\eta)$ and $h_{1}(\eta)$ in $(3.20)$ represent buoyant rotational flow driven by the lowest order temperature gradient; they decay exponentially in $\eta$. The flow terms with coefficient $A$ describe rotational flow (needed to satisfy no-slip at the interface) that decays exponentially in $\eta / P$, i.e., this describes the viscous boundary layer. The terms with coefficient $D$ represent a mass sink distributed along the positive $z$ axis whose strength increases linearly with $z$ (as $r \rightarrow 0$, $\left.\mathbf{u} \rightarrow-(2 z / r) \hat{\mathbf{r}}+\log \left(r^{2} / 4 z\right) \hat{\mathbf{z}}\right)$. The velocity from these terms grows as $\log \eta$, i.e., far away, the dendrite looks like a mass sink, to feed the rising, growing boundary layer. Finally, the flow proportional to $E$ is uniform flow $(\mathbf{u}=\hat{\mathbf{z}})$, which is needed to satisfy no penetration at the interface. So outside the thermal and viscous boundary layers, the perturbation flow becomes irrotational, as expected.

Using this flow solution, we can apply the same approach as above to solve the thermal problem $(3.7),(3.10),(3.12)$, and (3.13a) for the perturbations to the temperature $T_{1}$ and to the dendrite shape $H_{1}$. Assume solutions of the form

$$
\begin{aligned}
T_{1}(\xi, \eta) & =(\xi-1) p(\eta)+q(\eta), \\
H_{1}(\xi) & =H_{0}\left[(\xi-1) A_{0}+A_{1}\right],
\end{aligned}
$$


where $A_{0}$ and $A_{1}$ are constants to be determined. Then the problem separates to give

$$
\begin{aligned}
\left(\eta p^{\prime}\right)^{\prime}+\eta p^{\prime}-p & =-T_{0}^{\prime} g, \\
\left(\eta q^{\prime}\right)^{\prime}+\eta q^{\prime} & =-T_{0}^{\prime}\left(g+\frac{1}{2} h\right),
\end{aligned}
$$

with the boundary conditions

$$
\begin{aligned}
\text { at } \eta=H_{0}: \quad p & =-A_{0} T_{0}^{\prime}, \\
q & =-A_{1} T_{0}^{\prime}, \\
2 A_{0} & =-S\left[-A_{0} H_{0} T_{0}^{\prime}+p^{\prime}\right], \\
A_{1} & =-S\left[\left(\frac{1}{2} A_{0}-A_{1}\right) H_{0} T_{0}^{\prime}+q^{\prime}\right], \\
p & \rightarrow 0, \quad q \rightarrow 0,
\end{aligned}
$$

where $g(\eta)$ and $h(\eta)$ are the flow functions defined by (3.17), each consisting of several terms as shown in (3.20), and the identity $E_{1}^{\prime}+\eta E_{1}^{\prime \prime}=-\eta E_{1}^{\prime}$ was used to simplify the boundary conditions.

The general solution can be found by applying variation of parameters for each of the terms in $g$ and $h$, giving:

$$
\begin{aligned}
p(\eta)=\frac{2}{(P-1) E_{1}^{2}\left(H_{0}\right)}\left\{p_{1}(\eta)\right. & +A P p_{2}(\eta)+D p_{3}(\eta) \\
& \left.+J\left[E_{2}(\eta)-E_{1}(\eta)\right]+K[\eta+1]\right\}, \\
q(\eta)=\frac{1}{(P-1) E_{1}^{2}\left(H_{0}\right)}\left\{q_{1}(\eta)\right. & +A P q_{2}(\eta)+D q_{3}(\eta)+E q_{4}(\eta) \\
& \left.+L E_{1}(\eta)+M\right\},
\end{aligned}
$$

where $J, K, L, M$ are arbitrary constants and

$$
\begin{aligned}
p_{1}(\eta) & =\frac{1}{12}\left\{4\left[E_{2}(2 \eta)-2 E_{1}(2 \eta)\right]-3 E_{1}(\eta)\left[E_{2}(\eta)-E_{1}(\eta)\right]-2 e^{-\eta}\left[E_{2}(\eta)-2 E_{1}(\eta)\right]\right\} \\
p_{2}(\eta) & =\frac{1}{2}\left\{\left[E_{2}(\eta)-E_{1}(\eta)\right]\left[2 P E_{4}\left(\frac{\eta}{P}\right)-(1+2 P) E_{3}\left(\frac{\eta}{P}\right)+2 E_{2}\left(\frac{\eta}{P}\right)-E_{1}\left(\frac{\eta}{P}\right)\right]\right. \\
& \left.+\frac{1}{P}[\eta+1]\left[G_{22}(\eta)-G_{21}(\eta)-2 G_{12}(\eta)+2 G_{11}(\eta)+G_{02}(\eta)-G_{01}(\eta)\right]\right\} \\
p_{3}(\eta) & =-\left\{\left[E_{2}(\eta)-E_{1}(\eta)\right][1-\log \eta]+[\eta+1] E_{1}^{(2)}(\eta)\right\} \\
q_{1}(\eta) & =\frac{1}{2} E_{1}^{2}(\eta)+e^{-\eta} E_{1}(\eta)-2 E_{1}(2 \eta), \\
q_{2}(\eta) & =E_{1}(\eta)\left[(1-P) E_{3}\left(\frac{\eta}{P}\right)-(2-P) E_{2}\left(\frac{\eta}{P}\right)+E_{1}\left(\frac{\eta}{P}\right)\right] \\
& +\frac{1}{P}\left[(P-1) G_{21}(\eta)+(2-P) G_{11}(\eta)-G_{01}(\eta)\right] \\
q_{3}(\eta) & =2\left\{E_{1}(\eta)[1-\log \eta]+e^{-\eta}(\log \eta-2)-E_{1}^{(2)}(\eta)\right\} \\
q_{4}(\eta) & =-e^{-\eta},
\end{aligned}
$$


with the special functions $E_{1}^{(2)}$ and $G_{m n}$ defined by

$$
\begin{aligned}
E_{1}^{(2)}(\eta) & \equiv \int_{\eta}^{\infty} \frac{E_{1}(s)}{s} d s \\
G_{m n}(\eta) & \equiv \int_{\eta}^{\infty} E_{m}\left(\frac{s}{P}\right) E_{n}(s) d s
\end{aligned}
$$

Appendix A gives these functions in different forms that are computationally more convenient.

The boundary conditions (3.27-3.31) determine the constants. For the temperature to decay as $\eta \rightarrow \infty, K=M=0$. By eliminating $A_{0}$ and $A_{1}$ in the boundary conditions, we see that $J$ and $L$ are determined by

$$
\begin{aligned}
& H_{0} p^{\prime}\left(H_{0}\right)+\left(2+H_{0}\right) p\left(H_{0}\right)=0, \\
& H_{0} q^{\prime}\left(H_{0}\right)+\left(1+H_{0}\right) q\left(H_{0}\right)=-p\left(H_{0}\right) .
\end{aligned}
$$

The constants in the representation (3.24) of the shape perturbation $H_{1}$ are then given by

$$
\begin{aligned}
& A_{0}=S p\left(H_{0}\right) / H_{0} \\
& A_{1}=S q\left(H_{0}\right) / H_{0}
\end{aligned} .
$$

This completes the solution for the $\mathrm{O}(G)$ perturbation. (Note that at the Prandtl number $P=1$ the solution looks undefined, but the limit $P \rightarrow 1$ is well defined.)

The complicated form of the solution makes its interpretation difficult. Interpretation can be aided by considering the limits of small or large Peclet numbers $H_{0}$.

For large $H_{0}$, the relations given in Appendix A yield the following asymptotic forms:

$$
\begin{aligned}
& A_{0}=\frac{1}{4(P+1) H_{0}}-\frac{17 P+7}{8(P+1)^{2} H_{0}^{2}}+\mathrm{O}\left(H_{0}^{-3}\right) \\
& A_{1}=\frac{1}{4(P+1)}-\frac{11 P+9}{8(P+1)^{2} H_{0}}+\mathrm{O}\left(H_{0}^{-2}\right)
\end{aligned}
$$

In this limit, at leading order $A_{0}$ decays in $H_{0}$ and $A_{1}$ approaches a constant.

For small $H_{0}$, the asymptotic forms are far more complicated due to logarithmic singularities at the origin. The results are given at the end of Appendix A.

\section{RESULTS}

The above perturbation solution describes in detail the buoyant flow and the resulting thermal field around the dendrite tip. These solutions satisfy the correct interfacial conditions, but are only locally valid (Fig. 3); further up the dendrite the nonlinear effects of buoyant convection become significant, and far away from the dendrite the flow must depart from separable form to adjust to external conditions.

Two examples of the buoyant flow are illustrated in Figure 4. The interpretation of these vector fields depends on the reference frame. In the reference frame of the interface, 
each figure shows only the steady buoyant perturbation to the much larger uniform flow upward through the interface. Alternatively, in the reference frame of the immobile solid material of the dendrite, the interface moves downward at constant speed and so the flow, which is only that due to buoyancy, is unsteady. For the discussion below we adopt the latter viewpoint, in which each figure shows an instantaneous view of the entire (laboratoryframe) unsteady flow as the interface moves through.

In each case, the velocity vectors show the formation of a rising buoyant layer near the dendrite, which satisfies the no-slip condition at the solid surface. Farther away, the flow is drawn inward and downward to feed the growing buoyant layer. The temperature field decays over a dimensionless length scale of about unity because of the scaling; the viscous length scale is about $P$. (For very small dendrites, $H_{0} \ll 1$, these scales should be multiplied by $H_{0} \log H_{0}$ to account for the rapid variation of $T_{0}$ for small arguments.) In Figure 4a, $\left(H_{0}=1, P=2\right)$ the viscous and thermal length scales are comparable, and the local maximum of the vertical velocity in the buoyant layer occurs about 2 units from the dendrite. In Figure $4 \mathrm{~b},\left(H_{0}=0.1, P=0.1\right)$ the viscous length scale is much smaller than the thermal scale and this local maximum is much closer in, at a distance of about 0.3 units.

The buoyant layer flowing up the dendrite needs to be fed by incoming fluid. This fluid cannot come from far ahead of the dendrite, because the fluid far from the dendrite is not disturbed. Rather, it must come from the disturbed region outside the rising layer, yet still relatively close to the dendrite. The flow outside the buoyant layer has a downward component of velocity needed to conserve mass. To feed the beginnings of the buoyant layer at the tip, the incoming fluid approaching the axis must turn upward toward the tip. Consequently, a general feature of these flows is the presence of a dividing streamline (shown dashed) leading to a secondary stagnation point on the axis of symmetry below of the dendrite tip. In addition there is a primary stagnation point at the tip itself resulting from the no-penetration interface condition. Above the secondary stagnation point the inward flow turns up toward the dendrite tip, and below the secondary stagnation point the flow turns away from the tip. (Note that the above discussion refers to the perturbation streamlines, which satisfy continuity but are not the actual paths of fluid particles in this unsteady flow. As the interface and flow pattern sweep downward, fluid initially below the dividing streamline is later above it and ultimately is incorporated in the solid dendrite.)

There are two competing thermal effects of the secondary stagnation-point flow ahead of the tip. (i) When the stagnation point is outside the thermal boundary layer, the relatively cool fluid flowing upward toward the tip tends to steepen the thermal gradient and so promotes growth at the tip. (ii) When the stagnation point is well inside the thermal boundary layer, the warm fluid in the layer flowing laterally inward toward the axis (near the secondary stagnation point) acts to concentrate the heat directly ahead of the tip, and to flatten the the thermal gradient and hence decrease the growth. The position of the secondary stagnation point relative to the thermal boundary layer controls which of these two effects, vertical or horizontal convection of heat, will dominate. For a pure stagnation point flow, the vertical component increases with distance above the stagnation point whereas the lateral component is independent of height. As a result, when the secondary stagnation point is outside the thermal layer the vertical convection dominates; when 
the stagnation point is well inside the thermal boundary layer the horizontal convection dominates. Two simple examples of this competition are shown in Appendix B.

When the Prandtl number is relatively large, so is the viscous length scale, and the secondary stagnation point occurs far ahead of the tip, essentially outside the thermal layer. Then near the tip the vertical convection of heat dominates, compressing the gradient and enhancing the growth. This is the case in Figure 4a, where the secondary stagnation point is 2.3 units below the tip (outside the figure). For small $P$, however, the viscous layer is very thin, and the secondary stagnation point may occur very close to the tip, well inside the thermal boundary layer. In this case the horizontal convection of the heat within the boundary layer dominates, warming the fluid ahead of the tip and diminishing the growth, as in Figure 4b, where the stagnation point is only 0.285 units below the tip. Geometrical effects also play a role in the secondary stagnation point position; for a wide dendrite (large $\left.H_{0}\right)$ the stagnation point is fairly far from the tip even for small $P$.

The constants $A_{0}$ and $A_{1}$ describe how buoyancy changes the Peclet number $P e$, a measure of the growth at the tip, and modifies the shape of the dendrite. The interface position is given by:

$$
\eta=H(\xi) \sim H_{0}\left[1+G\left((\xi-1) A_{0}+A_{1}\right)\right]
$$

The Peclet number is based on the dimensional tip radius of curvature $R$ and growth speed $V:$

$$
P e \equiv R V / 2 \kappa \quad,
$$

which is the same as half the dimensionless tip radius. (This is the same definition for $P e$ used by Huang and Glicksman [2] and Horvay and Cahn [12]; Ananth and Gill's [3] $P e$ is twice ours.) Calculation of the curvature of the interface shape described by (4.1) at the tip, where $\xi=0$, then shows that the perturbed Peclet number is given by

$$
\begin{aligned}
P e & \sim H_{0}\left[1+G P e_{1}\right], \\
P e_{1} & \equiv\left(H_{0}-1\right) A_{0}+A_{1},
\end{aligned}
$$

where we have expanded $P e$ about its zero-buoyancy value $H_{0}$. Where the relative perturbation $P e_{1}>0$, buoyancy increases the Peclet number, which means growth at the tip is enhanced, and conversely, where $P e_{1}<0$, buoyancy diminishes growth at the tip.

The departure of the shape of the dendrite from a paraboloid depends only on $A_{0}$, as can be seen in (4.1), since for a paraboloidal dendrite the dimensionless interface position $H(\xi)$ would be a constant. Relative to the paraboloid corresponding to $A_{0}=0$, the shape modification is $H(\xi)-H_{0}\left(1+G A_{1}\right) \sim G H_{0} A_{0}(\xi-1)$. The results show that $A_{0}$ is always positive, which means that the tip $(\xi<1)$ is relatively smaller, but the dendrite widens further down $(\xi>1)$, because the buoyant flow redistributes the heat away from the tip towards the base of the dendrite. This widening means that locally the orientation of the interface is less vertical, so the local normal growth speed $V|\hat{\mathbf{z}} \cdot \hat{\mathbf{n}}|$ is increased.

The variations of the interface perturbations $A_{0}$ and $A_{1}$ and the Peclet number perturbation $P e_{1}$ with the parameters are shown in the next two figures. Figure 5 shows the dependence on the lowest order Peclet number $H_{0}$, which is monotonically related to the 
Stefan number $S$ (dimensionless undercooling) by the Ivantsov solution (3.4). As can be seen, over most of the parameter space $\left(H_{0}, P\right)$, buoyancy increases the Peclet number $\left(P e_{1}>0\right)$, and so the tip growth is enhanced by convection of released latent heat away from the tip (the exception is discussed below). For large undercooling (say, $H_{0}>1$, $S>0.6$ ) the perturbation $P e_{1}$ is relatively large, though in practice large $S$ means rapid solidification (large $V$ ), so $G \propto V^{-3}$ is small.

Comparing Figures 5a, 5b, 5c, and 5d shows that the value of the Prandtl number $P$ has a large effect on the magnitudes of $A_{0}$ and $A_{1}$, as well as some effect on their $H_{0}$-dependences. Figure 6 shows the dependence on Prandtl number $P$, for $H_{0}=1$ $(S=0.6$ ). For small $P$, the results become nearly independent of $P$, because the viscous layer near the interface is much thinner than the thermal layer, so that the buoyant flow is limited primarily by inertia. At the other extreme, when $P$ is large (for a given $G$ ), $A_{0}$ and $A_{1}$ apparently decrease roughly as $1 / P$. This follows from our scaling of the vorticity equation (2.11) based on inertia, rather than on viscosity. For large $P$, the flow is limited by viscosity throughout the thermal boundary layer, and the proper measure of the importance of buoyancy is the Rayleigh number $G / P$. Then to see the effects of $P$ on the perturbation, beyond this change of scale, we rescale $A_{0}$ and $A_{1}$ by $(1+P)$ in Figure $6 \mathrm{~b}$; this gives the correct scaling for large and small $P$. It is apparent that for large $P$ relative to small $P$, the rescaled $A_{1}$ is increased while $A_{0}$ is slightly decreased; as a result, $\mathrm{Pe}_{1}$ (rescaled) is increased.

Figure 5d shows that, for small $P$, there is a range of undercooling where buoyant convection reduces tip growth $\left(P e_{1}<0\right)$. The region in the parameter space where this occurs is shown in Figure 7 . The interpretation is that, when viscosity is relatively unimportant $(P \ll 1)$ and the dendrite is moderately small, the secondary stagnation point ahead of the dendrite occurs very near the tip, deep in the thermal boundary layer. Therefore, the lateral concentration of heat dominates the vertical convection near the tip and weakens the thermal gradient there, resulting in slower growth at the tip. For larger $P$, the viscous boundary layer becomes larger and the secondary stagnation point moves out of the thermal boundary layer; then near the tip the upward flow dominates the lateral flow and compresses the thermal gradient, leading to enhanced growth. Appendix B gives simple examples that illustrate how the stagnation-point flow can give opposite effects depending on the position of the secondary stagnation point. This distinction between small $P$ and not-small $P$ may give qualitatively different behavior for metals and organics.

The asymptotic approximations of the solution for large and small $H_{0}$ are compared with the actual solution (for $P=2$ ) in Figure 8. (At each extreme, both the leading-order approximation and the next higher-order approximation are shown.) It is apparent that the asymptotic forms converge well and so are useful.

Figure 9 shows how the Ivantsov solution is modified by buoyancy by giving the growth speed $V$ versus tip radius $R$, holding constant the undercooling, material properties (those of succinonitrile, given below), and gravity. This represents the family of possible physical solutions corresponding to one particular perturbation solution (fixed $S$ and $P$ ) for various $G$. Two cases are shown, differing only in Prandtl number; Figure 9a has $P=22.8$, that 
of succinonitrile, while Figure $9 \mathrm{~b}$ has $P=0.0441$. The form of the relation of $R$ and $V$ is

$$
R=\frac{A}{V}+\frac{B}{V^{4}}
$$

where $A=2 \kappa H_{0}$ and $B=2 g \alpha\left(T_{m}-T_{\infty}\right) \kappa^{2} H_{0} P e_{1}$. For the Ivantsov solution, $B=0$, giving the straight line of slope -1 on the logarithmic plot, and for large $V$ the solution with buoyancy is close to this line. However, for slow growth (small $V$, large buoyancy parameter $G$ ) the buoyancy causes departures from the Ivantsov solution. In Figure 9a $(P=22.8)$, buoyancy enhances tip growth and $B>0$, giving relatively faster growth for a given tip radius. In cases where buoyancy diminishes tip growth, then $B<0$, and the growth is slower for a given tip radius. In fact, the form of (4.4) gives an absolute maximum $R$ for negative $B$; Figure 9 b shows an example of reduced growth. Of course, any large departure from the Ivantsov solution violates the assumptions of the perturbation approximation, so the maximum in $R$ cannot be inferred from the present analysis.

A unique member of this family of solutions should be chosen using some selection criterion, which lies outside the scope of this paper. One criterion that seems to match experimental data well [2] comes from considering the morphological stability of the dendrite tip (with surface energy) in isolation; the criterion is of the form

$$
\frac{2 \kappa d_{0}}{V R^{2}}=\sigma^{*} \quad(\text { constant })
$$

where $d_{0} \equiv T_{m} \gamma c_{p} / \rho L^{2}$ is a capillary length, and $\gamma$ is the surface energy. Huang and Glicksman [2] review different theories that give slightly different values for the constant $\sigma^{*}$, but all of those values are close to the value that they determined experimentally using succinonitrile, which is $\sigma^{*}=0.0195$. This selection criterion is also shown (dotted) on Figures 9a and 9b. For modest or large $P$, as in Figure 9a, this criterion intersects the buoyancy-modified curve to give both faster growth and a smaller tip radius, relative to the non-buoyant (Ivantsov) case. The same selection criterion applied to a case where $P$ is small and buoyancy diminishes tip growth, as in Figure 9b, gives both slower growth and a larger tip. (In this case there may be two intersections, as shown in Figure 9b, and the upper should be chosen.) However, for some parameters there may be no intersection at all. Such cases involve buoyant effects strong enough to lie beyond the scope of this perturbation analysis. (Whether a maximum- $R$ condition can actually occur is an open question.)

The predictions of the buoyancy theory, augmented by the selection criterion $\sigma^{*}=$ 0.0195, are compared to the experiments of Huang and Glicksman [2] on succinonitrile in Figure 10. The values used for the physical constants are: $\kappa=1.16 \times 10^{-3} \mathrm{~cm}^{2} / \mathrm{s}, P=22.8$, $L=885.1 \mathrm{cal} / \mathrm{mole}, c_{p}=38.25 \mathrm{cal} / \mathrm{mole} \mathrm{K}, \alpha=5.0 \times 10^{-4} \mathrm{~K}^{-1}, d_{0}=2.7 \times 10^{-7} \mathrm{~cm}$, and $g=980 \mathrm{~cm} / \mathrm{s}^{2}$ (from Refs. $[2,6]$ ).

At high Stefan number $S$ (undercooling), buoyant convection is slow compared to the interface motion (the buoyancy parameter $G$ is small), and so buoyancy effects are negligible. As the Stefan number decreases, the growth speed $V$ also decreases, and the buoyancy parameter $G$ becomes significant. Both theory and experiments show that, relative to the non-buoyant case, buoyant convection increases the growth Peclet number $P e$, 
enhances the speed $V$ of growth, and decreases the radius $R$ of the dendrite tip. However, for very small undercooling, the theoretical predictions fail to match the experiments, overpredicting convective effects, due to neglecting the nonlinear inertial terms. Nonetheless, considering that the perturbation solution is based on the assumption that the buoyancy parameter is small, the range of agreement is surprisingly good (up to $G \approx 1000$ ).

\section{CONCLUSIONS}

The present work considers how buoyant convection affects an isolated axisymmetric dendrite of a pure substance, solidifying downward into an undercooled melt. Surface energy is neglected and thermodynamic equilibrium holds on the interface. The dimensionless problem depends on three parameters: the Stefan number $S=\left(T_{m}-T_{\infty}\right) c_{p} / L$ (undercooling), the Prandtl number $P$, and the buoyancy parameter $G=g \alpha \kappa\left(T_{m}-T_{\infty}\right) / V^{3}$. (Without surface energy, the problem has no length scale a priori, so the buoyancy parameter must involve either the size $R$ or growth speed $V$ of the dendrite.) Asymptotic representations are found for $S$ and $P$ fixed and $G \rightarrow 0$ giving local solutions for a region near the tip.

The buoyancy parameter $G$ is the product of a Rayleigh number (based on the thermal boundary layer thickness at the tip) and the Prandtl number $P$. For small $P$, inertia limits the flow and $G$ gives a dimensionless measure of the importance of buoyant convection. However, for large Prandtl number, viscosity limits the flow, and a better measure of the importance of buoyancy is the Rayleigh number $G / P$. One measure that covers both cases is $G /(P+1)$.

The solution is constructed as a regular perturbation in $G$ about the non-buoyant paraboloidal solution due to Ivantsov [1]. The order- $G$ perturbation quantities, describing the buoyant flow and temperature fields in the melt and the perturbed interface position, are given in closed form above, showing explicitly their dependences on $S$ and $P$. The perturbation solution is not uniformly valid in space; it applies within a dimensionless distance of $G^{-1} \gg 1$ from the dendrite tip, or in dimensional term $G^{-1} \kappa / V$.

A main result of interest is the dimensionless position of the solid-liquid interface in axisymmetric parabolic coordinates $(\xi, \eta)$. The interface is at $\eta=H(\xi)$, where

$$
H(\xi ; S, P, G) \sim H_{0}(S)\left\{1+G\left[(\xi-1) A_{0}(S, P)+A_{1}(S, P)\right]\right\} \quad,
$$

where $H_{0}$ is the Ivantsov solution, which increases monotonically with $S$ as given by (3.4), and the perturbations $A_{0}$ and $A_{1}$ are given by (3.37) and shown in Figures 5 and 6 . This result predicts two main effects of buoyant convection: enhanced or diminished tip growth, and a modified dendrite shape.

At the very tip of the dendrite, where $\xi=0$, the dimensionless radius of curvature is twice the Peclet number $P e=R V / 2 \kappa$, which measures the tip growth, and the relative $O(G)$ perturbation to $P e$ is defined by $(4.3)$ :

$$
P e_{1}=\left(H_{0}-1\right) A_{0}+A_{1}
$$

Thus, where $P e_{1}>0$ the Peclet number is increased by buoyancy effects, and tip growth is enhanced; this occurs for moderate to large Prandtl number $P>0.15$ or large undercooling 
$S>0.6$. For smaller $P$, however, there is a range of undercooling where $P e_{1}<0$, as shown in Figure 7, and in this parameter range the net effect of buoyant convection decreases the growth at the tip.

These differing effects at large and small $P$ can be explained in terms of the position of the secondary stagnation point on the axis ahead of the tip (as illustrated in Appendix B). When $P$ is large, the viscous length scale is larger than the thermal scale, and the stagnation point occurs outside the thermal boundary layer. Then the fluid flowing toward the tip is relatively cool, compressing the thermal gradient and enhancing growth. For small $P$, the viscous scale is small and the stagnation point can occur close to the tip, inside the thermal boundary layer. In that case, the lateral convection toward the axis around the secondary stagnation point tends to concentrate the heat of the boundary layer in the fluid ahead of the tip, thus reducing the vertical thermal gradient and diminishing growth. The shape of the dendrite also plays a role; for a wide dendrite (large $H_{0}$ and $S$ ), the stagnation point occurs outside the thermal boundary layer regardless of $P$.

While the zero-surface-energy solution cannot predict $R$ and $V$ separately, a selection criterion of the form $R^{2} V$-is-constant can be used. For the case of enhanced growth buoyant convection causes both faster growth and a smaller tip. For the case of diminished growth the same criterion will predict both slower growth and a larger tip.

The dependence of the dimensionless interface position $H$ on $\xi$ in (5.1) shows that the dendrite is no longer a paraboloid. The results show that $A_{0}>0$, so that toward the root the dendrite widens more quickly than a paraboloid of the same tip radius. This buoyancy modification of the dendrite shape is a unique feature of the current work; previous analyses assume a paraboloid shape.

For a given value of $G$, as the Prandtl number increases beyond unity the magnitude of these buoyant effects decreases roughly as $1 / P$, as shown in Figure 6a. This overall decrease is an artifact of the scaling. For large $P$, the thermal boundary layer is entirely within the viscous boundary layer, and so viscosity, not inertia, limits convection. A clearer picture of the effect of Prandtl number results from holding constant the importance of buoyancy, which is approximated by $G /(1+P)$; the rescaled results appear in Figure 6b. (The interpretation requires care, however, because $G$ is not directly subject to experimental control.)

Moreover, the character of the effect of buoyancy on tip growth changes with $P$; for large $P$ growth is always enhanced, while for small $P$ the growth at the tip may be reduced. Thus, conclusions based on experiments or calculations for a high-Prandtl number material (e.g., succinonitrile: $P=23$ ) should be applied cautiously to the processing of low-Prandtl number materials (e.g., metals).

The theory up to $G \approx 1000$ (with the selection criterion based on stability) compares well with the experimental results of Huang and Glicksman, as shown in Figure 10, and with the theory of Ananth and Gill [3]. However, due to the neglect of nonlinear inertial terms, the present work overpredicts buoyancy effects when $G$ is large. In contrast, the nonlinear calculations of Ananth and Gill [3] match the experiments well even for much larger $G$, as shown in their Figures 3 and 4 for $R$ and $V$ versus $S$; their selection criterion involves choosing the Grashof number for each value of $S$ to fit the experimental Peclet number. Their method, which assumes that the dendrite is a paraboloid, requires one to 
numerically solve a pair of coupled nonlinear ordinary differential equations for each set of parameters, and their published results are limited to a single Prandtl number $(P=23.1)$. Our work complements that of Ananth and Gill by considering a different parameter range, that of small $G$ rather than small $\xi$, and examining Prandtl number dependences and shape modifications. While our results are likely to be inaccurate for large $G$, the effects in that range may be qualitatively similar to our predictions.

The perturbation solution applies only for a downward growing dendrite, but since the perturbations to the order taken are linear in the buoyancy parameter $G$, it is tempting to apply the same solution to an upward growing dendrite by merely reversing the sign of $G$. We speculate that this may give reasonable results if the magnitude of $G$ is small enough, so that the speed of the interface as it sweeps through, freezing the material, overwhelms the instantaneous local fluid velocity. However, for larger buoyancy, i.e., slower solidification, the thermal layer of fluid rising toward the tip of the dendrite could keep rising past the tip, forming a plume above the dendrite. Such a plume would change the character of the flow completely, so the perturbation solution should not then apply.

The perturbation solution can also be interpreted to apply to the growth of a dendrite of a binary alloy, if the process is controlled by the mass diffusion of solute rejected at the solidification front (as discussed in Section 2). This would be the case if the mass diffusivity $D$ of the solute is much smaller than the thermal diffusivity $\kappa$ in the melt, and if the latent heat is negligible. Then the temperature is effectively constant $\left(T_{\infty}\right)$, and the transport problem reduces to convection and diffusion of solute, measured by its concentration $C$. The correspondence between the thermal and the solutal quantities is given in Section 2 .

The methods employed here may also be applicable to dendrites shaped more like elliptic paraboloids. The key element that makes the perturbation analysis feasible here is the existence of a simple solution to the nonbuoyant case, depending only on one coordinate in a separable coordinate system, that gives the shape of the free boundary and accounts for the nonlinear flux condition at the interface. The family of such solutions is quite extensive [15], including one for an elliptic paraboloid. (In fact, solutions are known for binary alloys when both thermal and solutal variations are important, or ternary alloys when thermal variations are negligible, so the perturbation approach may be applicable to such cases as well.) The detailed form of a buoyant perturbation to the elliptic-paraboloid state would be more complicated than the solution given here, in that the flow would depend on all three spatial coordinates, and the special functions may also be more complicated. We speculate that the qualitative features in that case may be similar to those of the present case.

\section{ACKNOWLEDGEMENTS}

This work was supported in part by a grant from the National Aeronautics and Space Administration, Microgravity Science and Applications Program, and in part by the Naval Postgraduate School Research Council. The authors are grateful to Dr. S. C. Huang for providing the experimental data cited, in tabular form.

\section{APPENDIX A: SPECIAL FUNCTIONS IN SOLUTION}


In the perturbation solution, the various functions ( $g$ 's, $h$ 's, $p$ 's, $q$ 's) describing the stream function and temperature perturbations are expressed in terms of exponential integrals $E_{n}$ and integrals thereof.

The exponential integrals are standard functions, and can be found in standard references, e.g., Ref. [14]. Some of their properties are recounted here, for convenience. The definition is

$$
E_{n}(x) \equiv \int_{1}^{\infty} \frac{e^{-x s}}{s^{n}} d s
$$

where we are only concerned with non-negative integer values for $n$. Some special cases are

$$
E_{0}(x)=\frac{e^{-x}}{x} \quad, \quad E_{1}(x)=\int_{x}^{\infty} \frac{e^{-s}}{s} d s
$$

Differentiating the definition shows that the derivatives are

$$
\frac{d}{d x} E_{n}(x)=-E_{n-1}(x)
$$

Integrating the definition by parts gives

$$
E_{n+1}(x)=\frac{1}{n}\left[e^{-x}-x E_{n}(x)\right]
$$

This can be used to reduce all such exponential integrals $E_{n}$ to forms involving only the special function $E_{1}$. At the origin, $E_{1}$ has a logarithmic singularity

$$
E_{1}(x)=-(\log x+\gamma)-\sum_{k=1}^{\infty} \frac{(-x)^{k}}{k k !}
$$

where $\gamma \approx 0.5772$ is Euler's constant. For large arguments, the asymptotic series is

$$
E_{1}(x) \sim \frac{e^{-x}}{x} \sum_{k=0}^{\infty} \frac{k !}{(-x)^{k}}
$$

The function $E_{1}^{(2)}$ is less common, but has applications to stellar atmospheres. It was first defined by van de Hulst [16]; we use his notation:

$$
E_{1}^{(2)}(x) \equiv \int_{x}^{\infty} \frac{E_{1}(s)}{s} d s
$$

Switching the order of integration, this can also be expressed as

$$
E_{1}^{(2)}(x)=\int_{1}^{\infty} \frac{e^{-x s} \log s}{s} d s
$$


which is more convenient for numerical calculation. (Ref. [17] shows this result and many others related to exponential integrals.) Near the origin, the following expansion, due to van de Hulst [16], converges quickly:

$$
E_{1}^{(2)}(x)=\frac{1}{2}(\log x+\gamma)^{2}+\frac{\pi^{2}}{12}+\sum_{k=1}^{\infty} \frac{(-x)^{k}}{k^{2} k !}
$$

For large arguments, integration by parts gives the following asymptotic series:

$$
E_{1}^{(2)}(x) \sim \frac{e^{-x}}{x} \sum_{k=1}^{\infty}\left[\frac{(-1)^{k-1} k !}{x^{k}} \sum_{j=1}^{k} \frac{1}{j}\right]
$$

(We have not seen this or subsequent results published previously.)

Van de Hulst [16] defined certain functions he called $G_{m n}$; the functions we call $G_{m n}$ are similar to, but different from, his functions. We use the definition

$$
G_{m n}(x) \equiv \int_{x}^{\infty} E_{m}\left(\frac{s}{P}\right) E_{n}(s) d s,
$$

where the parameter $P$ is the Prandtl number for our purposes. Following the methods used by Chandrasekhar [18], i.e., integration by parts and the identities for $E_{n}$, all of the $G_{m n}$ we need can be reduced to simpler functions:

$$
\begin{aligned}
G_{02}(x) & =-P^{2} e^{-x / P} E_{1}(x)+\left(P+P^{2}\right) E_{1}(\beta x), \\
G_{11}(x) & =e^{-x} E_{1}(x / P)+P e^{-x / P} E_{1}(x)-x E_{1}(x) E_{1}(x / P)-(P+1) E_{1}(\beta x), \\
G_{12}(x) & =\frac{1}{2}\left[P e^{-\beta x}+(1-x) e^{-x} E_{1}(x / P)-\left(P^{2}+P x\right) e^{-x / P} E_{1}(x)\right. \\
& \left.+x^{2} E_{1}(x) E_{1}(x / P)+\left(P^{2}-1\right) E_{1}(\beta x)\right], \\
G_{21}(x) & =\frac{1}{2}\left[e^{-\beta x}-\frac{1}{P}(1+x) e^{-x} E_{1}(x / P)+(P-x) e^{-x / P} E_{1}(x)\right. \\
& \left.+\frac{x^{2}}{P} E_{1}(x) E_{1}(x / P)-\left(P-\frac{1}{P}\right) E_{1}(\beta x)\right], \\
G_{22}(x) & =\frac{1}{3}\left[(P+1-x) e^{-\beta x}-\frac{1}{P}\left(1+x-x^{2}\right) e^{-x} E_{1}(x / P)-\right. \\
& \left.-\left(P^{2}+P x-x^{2}\right) e^{-x / P} E_{1}(x)-\frac{x^{3}}{P} E_{1}(x) E_{1}(x / P)+\left(P^{2}+\frac{1}{P}\right) E_{1}(\beta x)\right],
\end{aligned}
$$

where $\beta \equiv 1+\frac{1}{P}$. The one exception is $G_{01}$, which apparently cannot be reduced to standard functions. By definition,

$$
\frac{1}{P} G_{01}(x)=\int_{x}^{\infty} \frac{e^{-s / P}}{s} E_{1}(s) d s .
$$


Switching the order of integration yields a form more amenable to numerical computation:

$$
\frac{1}{P} G_{01}(x)=\int_{\beta x}^{\infty} \frac{e^{-s}}{s} \log \left(\frac{s}{x}-\frac{1}{P}\right) d s .
$$

Near the origin, the series representation is

$$
\begin{aligned}
\frac{1}{P} G_{01}(x) & =\frac{1}{2}(\log x+\gamma)^{2}-\frac{\pi^{2}}{12}-\log ^{2} P-\operatorname{dil} \log (P+1) \\
& +(-(\log x+\gamma)-P+1) \frac{x}{P}+\left((\log x+\gamma)+\frac{1}{2}\left(P^{2}+4 P-1\right)\right) \frac{x^{2}}{4 P^{2}} \\
& +\left(-(\log x+\gamma)-\frac{1}{6}\left(2 P^{3}+9 P^{2}+18 P-1\right)\right) \frac{x^{3}}{18 P^{3}}+\mathrm{O}\left(x^{4} \log x\right),
\end{aligned}
$$

where the dilogarithm function is defined by

$$
\operatorname{dilog}(x) \equiv \int_{x}^{1} \frac{\log (s)}{s-1} d s
$$

following Abramowitz and Stegun [14]. (The general form for the $n^{\text {th }}$ term in (A.15) is not apparent.) Integration by parts gives the asymptotic series for large arguments:

$$
\frac{1}{P} G_{01}(x) \sim \frac{P e^{-\beta x}}{x} \sum_{k=1}^{\infty}\left[\frac{(-1)^{k-1} k !}{x^{k}} \sum_{j=1}^{k} \frac{1}{j \beta^{k-j+1}}\right]
$$

Using the reductions above, all the perturbation functions ( $g$ 's, $h$ 's, $p$ 's, $q$ 's) can be expressed in forms where the only special functions are $E_{1}, E_{1}^{(2)}$, and $G_{01}$ :

$$
\begin{aligned}
g_{1}(x) & =\frac{1}{2}\left[(1+x) e^{-x}-\left(2 x+x^{2}\right) E_{1}(x)\right] \\
h_{1}(x) & =-x e^{-x}+\left(x+x^{2}\right) E_{1}(x) \\
p_{1}(x) & =\frac{1}{12}\left\{2 e^{-2 x}+(1+2 x) e^{-x} E_{1}(x)+(1+x)\left[3 E_{1}^{2}(x)-8 E_{1}(2 x)\right]\right\}, \\
p_{2}(x) & =\frac{1}{12}\left\{-\frac{2 P^{2}-5 P+1}{P} e^{-\beta x}+\left(\frac{-6 P^{2}+6 P+1}{P^{2}}+\frac{2}{P^{2}} x\right) e^{-x} E_{1}(x / P)\right. \\
& \left.+(1+x)\left[6\left(E_{1}(x) E_{1}(x / P)-\frac{1}{P} G_{01}(x)\right)+\frac{2 P^{3}-3 P^{2}-6 P-1}{P^{2}} E_{1}(\beta x)\right]\right\} \\
p_{3}(x) & =(\log x-1) e^{-x}-(1+x)\left[(\log x-1) E_{1}(x)+E_{1}^{(2)}(x)\right] \\
q_{1}(x) & =\frac{1}{2}\left\{2 e^{-x} E_{1}(x)+\left[E_{1}^{2}(x)-4 E_{1}(2 x)\right]\right\} \\
q_{2}(x) & =\frac{1}{2}\left\{\frac{P-1}{P} e^{-\beta x}+\left(\frac{-2 P^{2}+3 P+1}{P^{2}}-\frac{P^{2}-1}{P^{2}} x\right) e^{-x} E_{1}(x / P)\right. \\
& \left.+\left[2\left(E_{1}(x) E_{1}(x / P)-\frac{1}{P} G_{01}(x)\right)+\frac{P^{3}-P^{2}-3 P-1}{P^{2}} E_{1}(\beta x)\right]\right\} \\
q_{3}(x) & =2\left\{(\log x-2) e^{-x}-\left[(\log x-1) E_{1}(x)+E_{1}^{(2)}(x)\right]\right\}
\end{aligned}
$$


To calculate these functions, standard mathematical software libraries have built-in functions for the exponential integral and routines for numerically evaluating integrals such as (A.8) and (A.14), which define $E_{1}^{(2)}$ and $G_{01}$. For extreme values of the arguments, the appropriate series for $E_{1}^{(2)}$ and $G_{01}$ may be more accurate than numerical integration.

Using the small-argument forms for these functions gives the asymptotic forms of the coefficients in the shape perturbation (3.24) for small $H_{0}$. Because of the logarithmic terms, these asymptotic expressions are given as quotients: 


$$
\begin{aligned}
A_{0} & =2\left\{\operatorname { l o g } ( H _ { 0 } ) \left[\frac{2}{3} \log (2)-\frac{P}{2} \operatorname{dilog}(P+1)+\log (P+1)\left(\frac{P^{2}}{6}-\frac{P}{4}-\frac{1}{2}-\frac{1}{12 P}\right)\right.\right. \\
& \left.-\frac{P}{4} \log ^{2}(P)+\log (P)\left(\frac{-P^{2}}{6}+\frac{P}{4}\right)-\frac{P \pi^{2}}{12}+\frac{\pi^{2}}{24}-\frac{P}{6}+\frac{1}{6}\right] \\
& +\left[-\frac{2}{3} \log (2) \log (P)+\frac{2}{3} \log (2)(\gamma+1)-\frac{P}{2} \operatorname{dilog}(P+1)(\gamma+1)\right. \\
& +\log (P+1)(\gamma+1)\left(\frac{P^{2}}{6}-\frac{P}{4}-\frac{1}{2}-\frac{1}{12 P}\right)-\frac{P}{4} \log ^{2}(P)(\gamma+1) \\
& \left.\left.+\log (P)\left((\gamma+1)\left(-\frac{P^{2}}{6}+\frac{P}{4}\right)-\frac{\pi^{2}}{24}-\frac{1}{12}\right)+(\gamma+1)\left((-2 P+1) \frac{\pi^{2}}{24}-\frac{P-1}{6}\right)\right]+\mathrm{O}\left(H_{0} \log ^{2}\left(H_{0}\right)\right)\right\} / \\
& (P-1)\left\{-2 \log ^{3}\left(H_{0}\right)+\log ^{2}\left(H_{0}\right)[2 \log (P)-6 \gamma-5]+\log \left(H_{0}\right)\left[\log (P)(4 \gamma+3)-6 \gamma^{2}-10 \gamma-3\right]\right. \\
& \left.+\left[(\log (P)-\gamma-1)\left(2 \gamma^{2}+3 \gamma\right)\right]+\mathrm{O}\left(H_{0} \log ^{3}\left(H_{0}\right)\right)\right\}
\end{aligned}
$$

$$
\begin{aligned}
A_{1} & =\left\{\operatorname { l o g } ^ { 2 } ( H _ { 0 } ) \left[-\frac{8}{3} \log (2)+P \operatorname{dil} \log (P+1)+\log (P+1)\left(\frac{-2 P^{2}}{3}+\frac{P}{2}+2+\frac{5}{6 P}\right)\right.\right. \\
& \left.+\frac{P}{2} \log ^{2}(P)+\log (P)\left(\frac{2 P^{2}}{3}-\frac{P}{2}\right)+(2 P-1) \frac{\pi^{2}}{12}+\frac{2}{3}(P-1)\right] \\
& +\log \left(H_{0}\right)\left[\frac{8}{3} \log (2) \log (P)-\frac{2}{3} \log (2)(8 \gamma+13)+P \operatorname{dil} \log (P+1)(2 \gamma+4)\right. \\
& +\log (P+1)\left(\frac{-4 P^{2} \gamma}{3}+P \gamma+4 \gamma+\frac{5 \gamma}{3 P}-\frac{13 P^{2}}{6}+2 P+\frac{13}{2}+\frac{7}{3 P}\right)+P \log ^{2}(P)(\gamma+2) \\
& \left.+\log (P)\left(\frac{\pi^{2}}{12}+\frac{4 P^{2} \gamma}{3}-P \gamma+\frac{13 P^{2}}{6}-2 P-\frac{1}{6}\right)+(2 P-1)(\gamma+2) \frac{\pi^{2}}{6}+(P-1)\left(\frac{4 \gamma}{3}+\frac{13}{6}\right)\right] \\
& +\left[\log (2) \log (P)\left(\frac{8 \gamma}{3}+6\right)+\log (2)\left(\frac{-8 \gamma^{2}}{3}-\frac{26 \gamma}{3}-6\right)+P \operatorname{dilog}(P+1)\left(\gamma^{2}+4 \gamma+3\right)\right. \\
& +\log (P+1)\left(\frac{-2 P^{2} \gamma^{2}}{3}+\frac{P \gamma^{2}}{2}+2 \gamma^{2}+\frac{5 \gamma^{2}}{6 P}-\frac{13 P^{2} \gamma}{6}+2 P \gamma+\frac{13 \gamma}{2}+\frac{7 \gamma}{3 P}-\frac{3 P^{2}}{2}+\frac{3 P}{2}+\frac{9}{2}+\frac{3}{2 P}\right) \\
& +\frac{P}{2} \log (P)\left(\gamma^{2}+4 \gamma+3\right) \\
& +\log (P)\left(\frac{\pi^{2} \gamma}{12}+\frac{\pi^{2}}{4}+\frac{2 P^{2} \gamma^{2}}{3}-\frac{P \gamma^{2}}{2}+\frac{13 P^{2} \gamma}{6}-2 P \gamma-\frac{\gamma}{6}+\frac{3 P^{2}}{2}-\frac{3 P}{2}\right) \\
& \left.\left.+(2 P-1)\left(\gamma^{2}+4 \gamma+3\right) \frac{\pi^{2}}{12}+(P-1)\left(\frac{2 \gamma^{2}}{3}+\frac{13 \gamma}{6}+\frac{3}{2}\right)\right]+\mathrm{O}\left(H_{0} \log ^{3}\left(H_{0}\right)\right)\right\} / \\
+ & P-1)\left\{2 \log \left(H_{0}\right)+\log ^{3}\left(H_{0}\right)[-2 \log (P)+8 \gamma+7]+\log ^{2}\left(H_{0}\right)\left[-\log ^{2}(P)(6 \gamma+5)+12 \gamma^{2}+21 \gamma+8\right]\right. \\
+ & \log \left(H_{0}\right)\left[-\log (P)\left(6 \gamma^{2}+10 \gamma+3\right)+8 \gamma^{3}+21 \gamma^{2}+16 \gamma+3\right] \\
+ & {\left.\left.[\log (P)+\gamma+1)\left(2 \gamma^{3}+5 \gamma^{2}+3 \gamma\right)\right]+\mathrm{O}\left(H_{0} \log ^{4}\left(H_{0}\right)\right)\right\} }
\end{aligned}
$$


where $\gamma$ is Euler's constant $(0.5772 \ldots)$. (The lengthy algebraic calculations preceding these results were verified using the symbolic mathematics program Mathematica.)

\section{APPENDIX B: EFFECT OF STAGNATION POINT POSITION}

A simplified two-dimensional problem illustrates how the position of the stagnation point ahead of the dendrite affects the thermal gradient at the tip.

Consider a planar interface moving in the positive $z$ direction at constant speed unity, as shown in Figure 11. In coordinates moving with the front there is a unit uniform velocity in the $-z$ direction. In dimensionless form, the temperature $T$ is governed by the convection-diffusion equation in Cartesian coordinates $(x, z)$

$$
\begin{aligned}
T_{z z}+T_{x x} & =(-1+w) T_{z}+u T_{x}, \\
\text { at } z=0: \quad T & =1, \\
\text { as } z \rightarrow \infty: \quad T & \rightarrow 0,
\end{aligned}
$$

where $(u, w)$ is the velocity field imposed on the system; the velocity satisfies the continuity equation

$$
u_{x}+w_{z}=0
$$

When no flow is imposed, there is a one-dimensional conduction solution,

$$
T(z)=e^{-z}
$$

(This planar solution fails to satisfy the energy-flux condition (2.15) except for the special undercooling $S=1$.) The thermal boundary layer has no definite edge, but for this discussion we define the boundary-layer thickness to be unity (so $T=0.37$ at the edge).

With an imposed flow, the temperature is still one-dimensional, viz. $T(z)$, provided that $w=w(z)$ only. Then the system reduces to

$$
\begin{aligned}
T_{z z}+T_{z} & =w(z) T_{z} \\
T(0) & =1, \\
T(\infty) & =0 .
\end{aligned}
$$

The convection term $-w(z) T_{z}$ acts as a local heat sink where the fluid flows in the same direction as the temperature gradient ( $w$ and $T_{z}$ are of the same sign). Conversely, the convection term gives a local heat source where the fluid flows in the direction opposite to the thermal gradient. Note that this thermal problem is unaffected by the horizontal component of velocity (which is $u=-x w_{z}$ by continuity); there is no net horizontal transfer of heat.

To determine the thermal gradient at the front, integrate the first of (B.4) from zero to infinity, use the boundary conditions and integration by parts, to obtain

$$
T_{z}(0)=-1-\int_{0}^{\infty} w T_{z} d z
$$


On the right-hand side of (B.5), the first term represents the gradient due to pure conduction (see equation (B.3)), the second, the cumulative heating/cooling due to vertical convection. If the temperature is decreasing with $z$ and the vertical flow is upward everywhere (so the $w T_{z}>0$ ), then the gradient at the interface is steepened by convection, because locally the convection has the effect of a heat sink at each point. But if the flow is downward in some places and upward in others, then at the interface the net effect could be of either sign; convection can either steepen or flatten the gradient. This effect of convection on the gradient at the interface, being cumulative in nature, is a nonlocal effect.

\section{Example I}

Let the conduction solution (B.3) be slightly perturbed by imposing a weak, stagnationpoint flow a fixed distance $z_{s}$ ahead of the interface:

$$
(u, w)=\epsilon\left(-x, z-z_{s}\right) \quad, \quad 0<\epsilon \ll 1 \quad,
$$

as shown in Figure 11. (For simplicity we do not worry about satisfying the flow boundary conditions at the interface.) We express the resulting temperature in powers of $\epsilon$ as follows:

$$
T=T_{0}+\epsilon T_{1}+\cdots
$$

The leading-order temperature is the conduction solution,

$$
T_{0}=e^{-z}
$$

The first correction satisfies

$$
\begin{aligned}
T_{1 z z}+T_{1 z} & =\left(z-z_{0}\right) T_{0 z} \\
T_{1}(0) & =0 \\
T_{1}(\infty) & =0
\end{aligned}
$$

The solution is easily found to be

$$
T_{1}=\left[\left(1-z_{s}\right) z+\frac{z^{2}}{2}\right] e^{-z}
$$

The thermal gradient at the interface thus has the form

$$
T_{z}(0) \sim-1+\epsilon\left(1-z_{s}\right)
$$

If the stagnation point is outside the thermal boundary layer, $z_{s}>1$,

$$
\left|T_{z}(0)\right| \sim 1+\epsilon\left|1-z_{s}\right|
$$

and the gradient at the interface is steepened, because the convection toward the interface, where the gradient is strongest, cools the fluid and more than compensates for the small 
heating due to the convection away from the interface out where the gradient is weak. If the stagnation point is inside the boundary layer, $z_{s}<1$,

$$
\left|T_{z}(0)\right| \sim 1-\epsilon\left|1-z_{s}\right|
$$

and the gradient is eased because the cumulative heating from the downward flow nearby, where the gradient is still significant, overcomes the cooling from the upward flow above the stagnation point.

While this highly simplified result does not apply directly to the dendrite analysis, it does show how flow whose vertical velocity component takes on both signs can affect the growth of the tip in two different ways. A secondary stagnation point inside the thermal boundary layer can weaken the thermal gradient at the interface, and thus diminish the growth, while for a secondary stagnation point further away, outside the boundary layer, the effects are reversed.

\section{Example II}

To better approximate the flow near the dendrite tip, let the weak imposed flow satisfy the no-slip, no-penetration conditions at the interface, as well as the linearized vorticity equation

$$
-\omega_{z}=P \nabla^{2} \omega
$$

where $\omega=u_{z}-w_{x}$ is teh $y$ ccompnoent of vorticity and $P$ is the Prandtl number. We choose a flow of the form

$$
\begin{aligned}
(u, w) & =\epsilon\left(-x f^{\prime}(z), f(z)\right), \\
f(z) & \equiv-e^{-z / P}+1-\frac{z}{P}+\frac{b}{2}\left(\frac{z}{P}\right)^{2},
\end{aligned}
$$

which includes a secondary stagnation point $\left(f\left(z_{s}\right)=0\right)$ whose position $z_{s}$ ranges from infinity to zero as the parameter $b$ varies from 0 to 1 . (Note that $w(0)=f(0)=0$ and $u(x, 0)=-x f^{\prime}(0)=0$, and so there is also a primary stagnation point at the origin. As fluid flows toward the interface, for $0<z<z_{s}$, the horizontal component of velocity is at first inward, toward the $z$ axis, near the secondary stagnation point, and then outward near the interface.)

Then the thermal gradient at the interface is given by (B.5), appropriately linearized:

$$
\begin{aligned}
T_{z}(0) & =-1+\epsilon \int_{0}^{\infty}-f^{\prime}(z) T_{0}(z) d z \\
& =-1+\epsilon\left(\frac{b}{P^{2}}-\frac{1}{P(P+1)}\right) .
\end{aligned}
$$

This means that the net effect of the lateral convection of heat as the fluid flows toward the interface depends on $b$, and thus on the position of the secondary stagnation point. If $b<P /(P+1)$ then the stagnation point is relatively far away, outside the thermal boundary layer, and the effect is to cool the fluid flowing toward the interface, strengthening the thermal gradient. Conversely, if $b>P /(P+1)$, the stagnation point is close to or inside 
the thermal boundary layer, and the net effect of vertical flow is to heat the fluid flowing toward the interface, weakening the gradient.

For example, if one chooses $P=1$, the thermal effect reverses at $b=\frac{1}{2}$, which corresponds to the secondary stagnation point location $z_{s}=2.56$, where the temperature is $T_{0}=0.08$. (As $P$ varies from zero to infinity, the stagnation point position at which there is no enhancement of the thermal gradient moves from $z_{s}=2$ to $z_{s}=3$.) When the secondary stagnation point is closer than this, the thermal gradient is enhanced; when farther away, the gradient is diminished.

\section{REFERENCES}

1. G. P. Ivantsov, Dokl. Akad. Nauk, SSSR. 58, 567 (1947).

2. S. C. Huang and M. E. Glicksman, Acta metall. 29, 701 (1981).

3. R. Ananth and W. N. Gill, J. Cryst. Growth 91, 587 (1988).

4. F. E. Rosenberger, Fundamentals of Crystal Growth I, p. 2. Springer-Verlag (1979).

5. W. Kurz and D. J. Fisher, Fundamentals of Solidification. Trans Tech Publications (1986).

6. S. C. Huang and M. E. Glicksman, The influence of natural convection on dendritic growth, Technical Report 81CRD287, General Electric Company, Corporate Research and Development (1981).

7. S. H. Tirmizi and W. N. Gill, J. Cryst. Growth 85, 488 (1987).

8. W. W. Mullins and R. F. Sekerka, J. appl. Phys. 35, 444 (1964).

9. W. Oldfield, Mater. Sci. Engng. 11, 211 (1973).

10. J. S. Langer and H. Muller-Krumbhaar, Acta metall. 26, 1681 (1978).

11. R. D. Doherty, B. Cantor and S. Fairs, Metall. Trans. A 9, 621 (1978).

12. G. Horvay and J. W. Cahn, Acta metall. 9, 695 (1961).

13. F. S. Ham, Quart. Appl. Math. 17, 137 (1959).

14. M. Abramowitz and I. A. Stegun, Handbook of Mathematical Functions. Dover Publi- 
cations (1972).

15. D. Canright and S. H. Davis, Metall. Trans. A 20, 225 (1989).

16. H. C. van de Hulst, Astrophys. J. 107, 220 (1948).

17. V. Kourganoff, Basic methods in transfer problems, App. I. Oxford University Press (1952).

18. S. Chandrasekhar, Radiative Transfer. Oxford University Press (1950). 
Figures for "Buoyancy Effects of a Growing, Isolated Dendrite"

David Canright

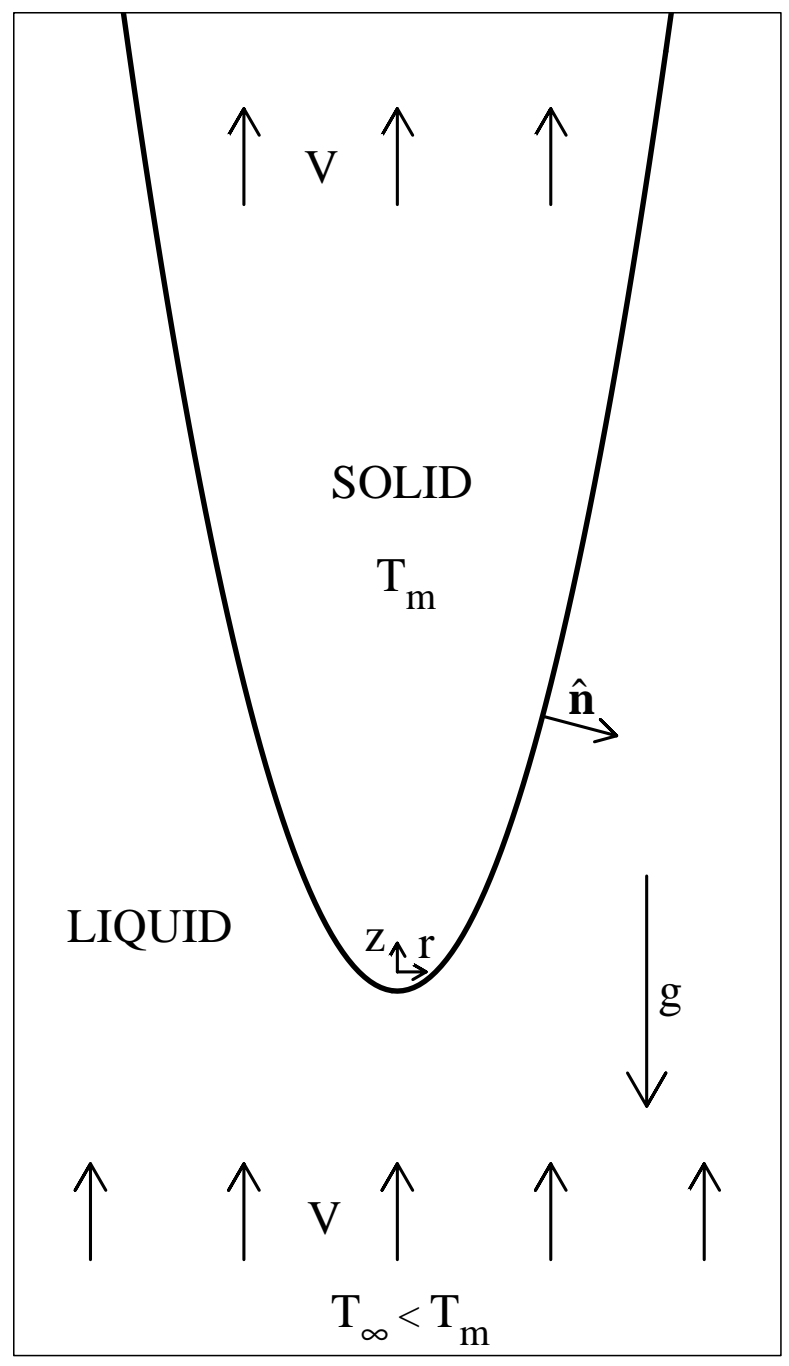

Figure 1: Problem schematic: Relative to the growing dendrite surface, the liquid moves steadily up and through the interface as it becomes solid. Axisymmetry is assumed and gravity is directed downward. Far from the dendrite the liquid is undercooled and in uniform motion. 


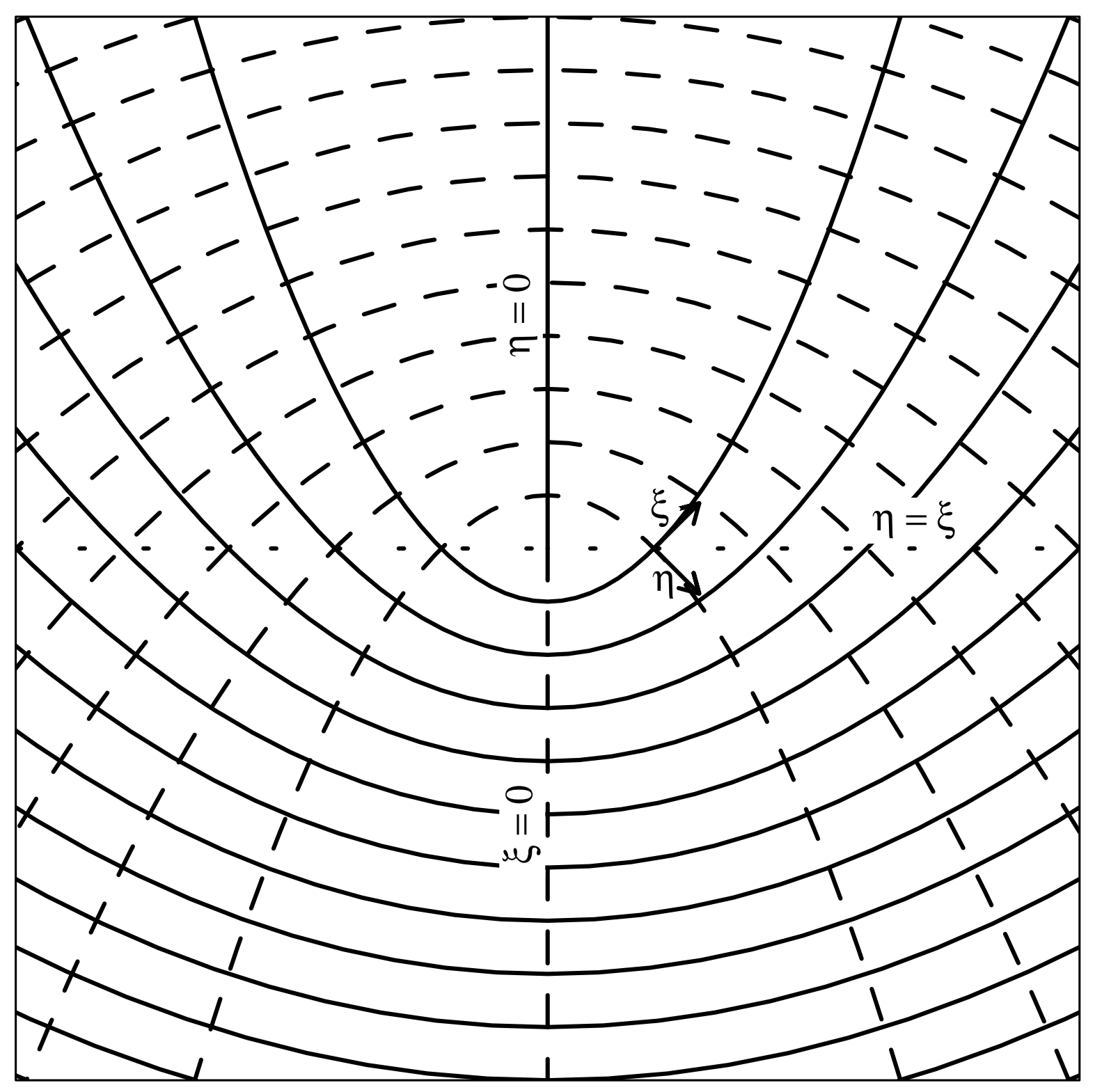

Figure 2: Parabolic coordinates: Lines of constant $\eta$ (solid) and $\xi$ (dashed) are shown; equal steps in $\eta$ and $\xi$ correspond to equal distances along the $z$-axis. 


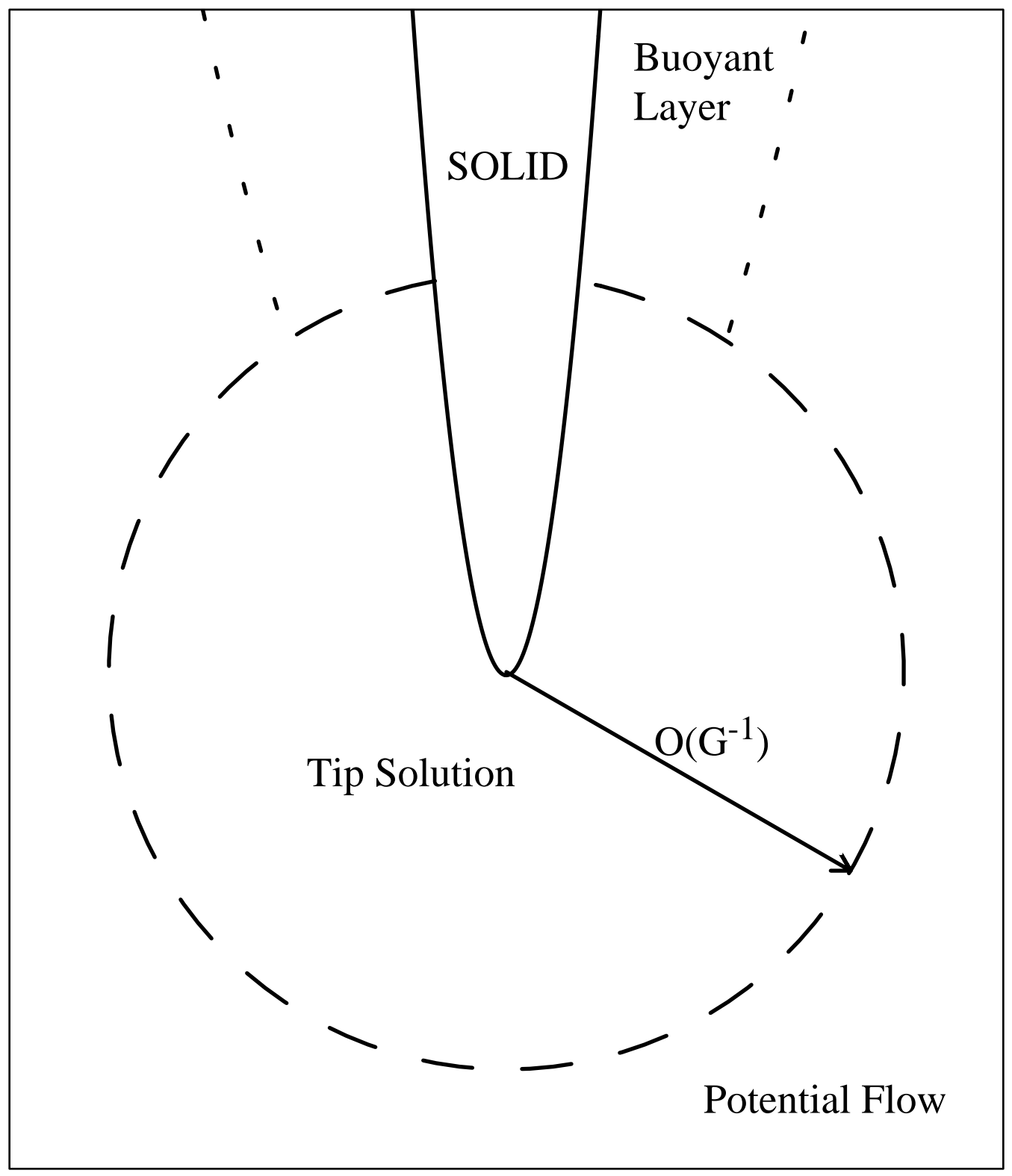

Figure 3: Regions of flow: The perturbation solution applies within a distance $\mathrm{O}\left(G^{-1}\right)$ from the dendrite tip. In the buoyant layer further along the dendrite convection is strongly nonlinear. In the far field the flow is irrotational but not of separable form. 


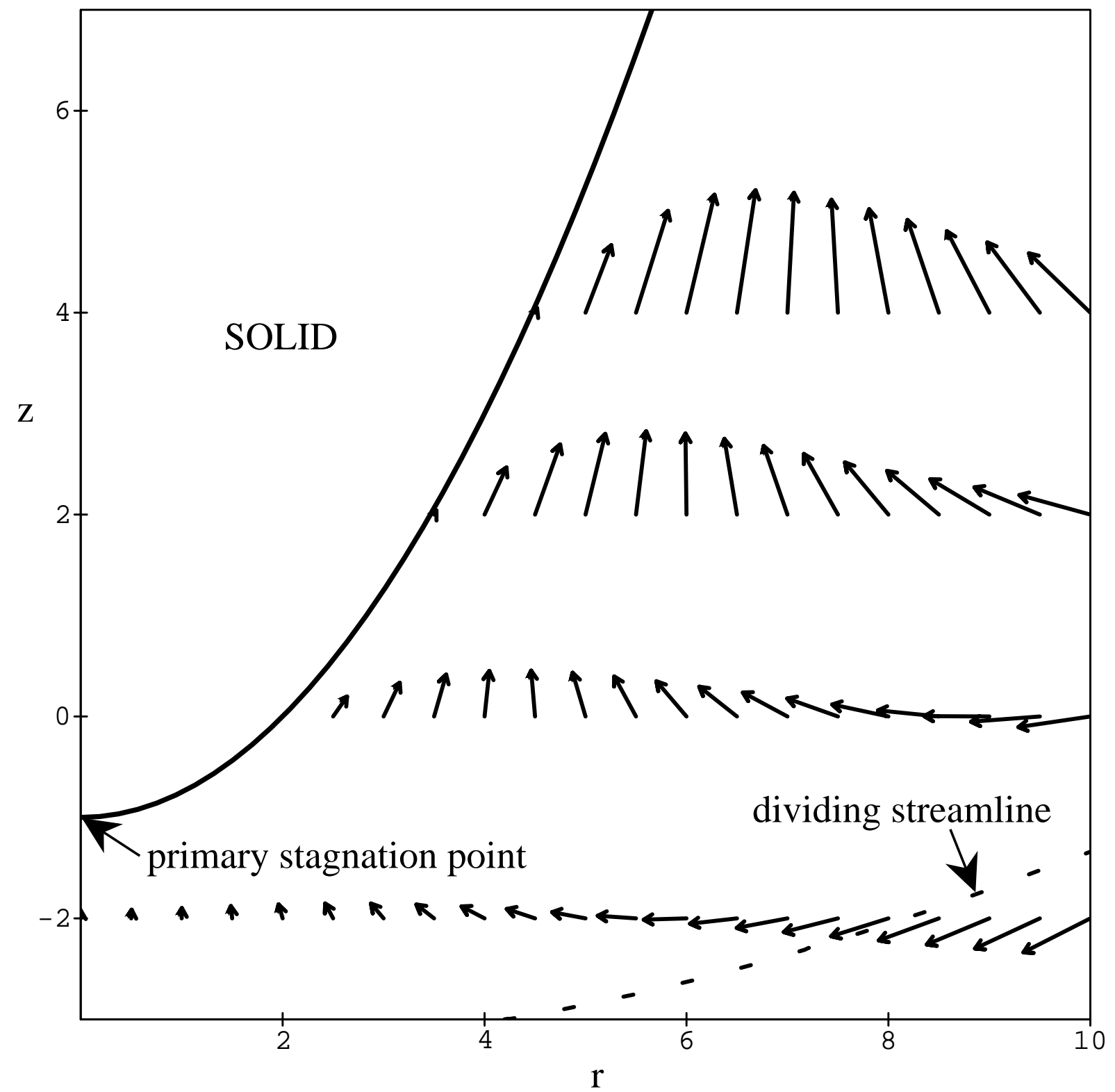

Figure 4: Buoyant flow: two examples of the instantaneous flow field (velocity vectors) near the dendrite tip, relative to the solid material (the interface moves downward at speed $V$ in the laboratory frame). The dashed line shows the streamline (not a particle path) to the stagnation point on the axis of symmetry, which divides fluid turning upward toward the tip from that flowing downward away from the tip. (a) $H_{0}=1$ $(S=0.6), P=2$, stagnation point at $z=-3.31$. 


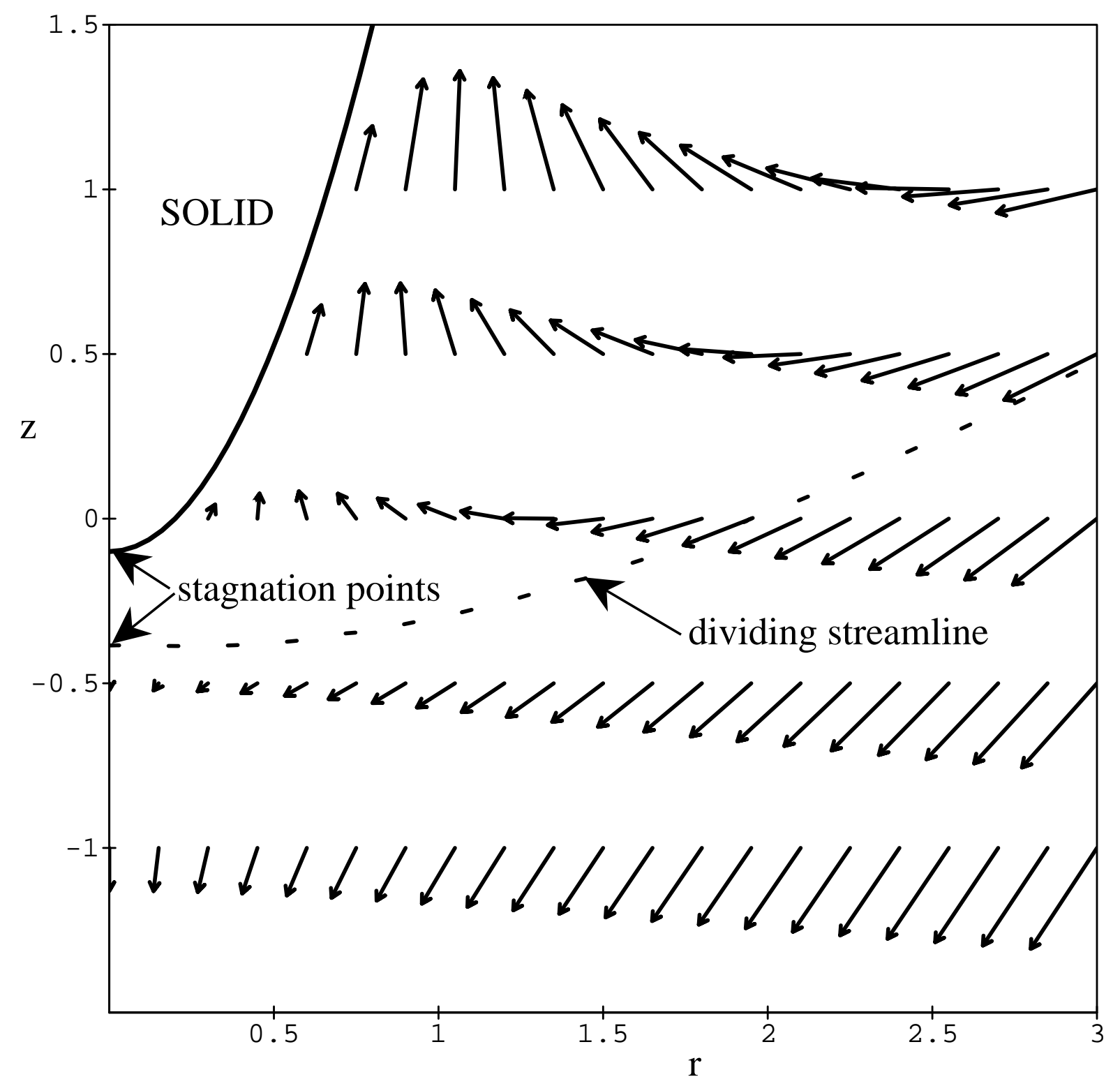

Figure 4: (b) $H_{0}=0.1(S=0.2), P=0.1$, stagnation point at $z=-0.385$. 


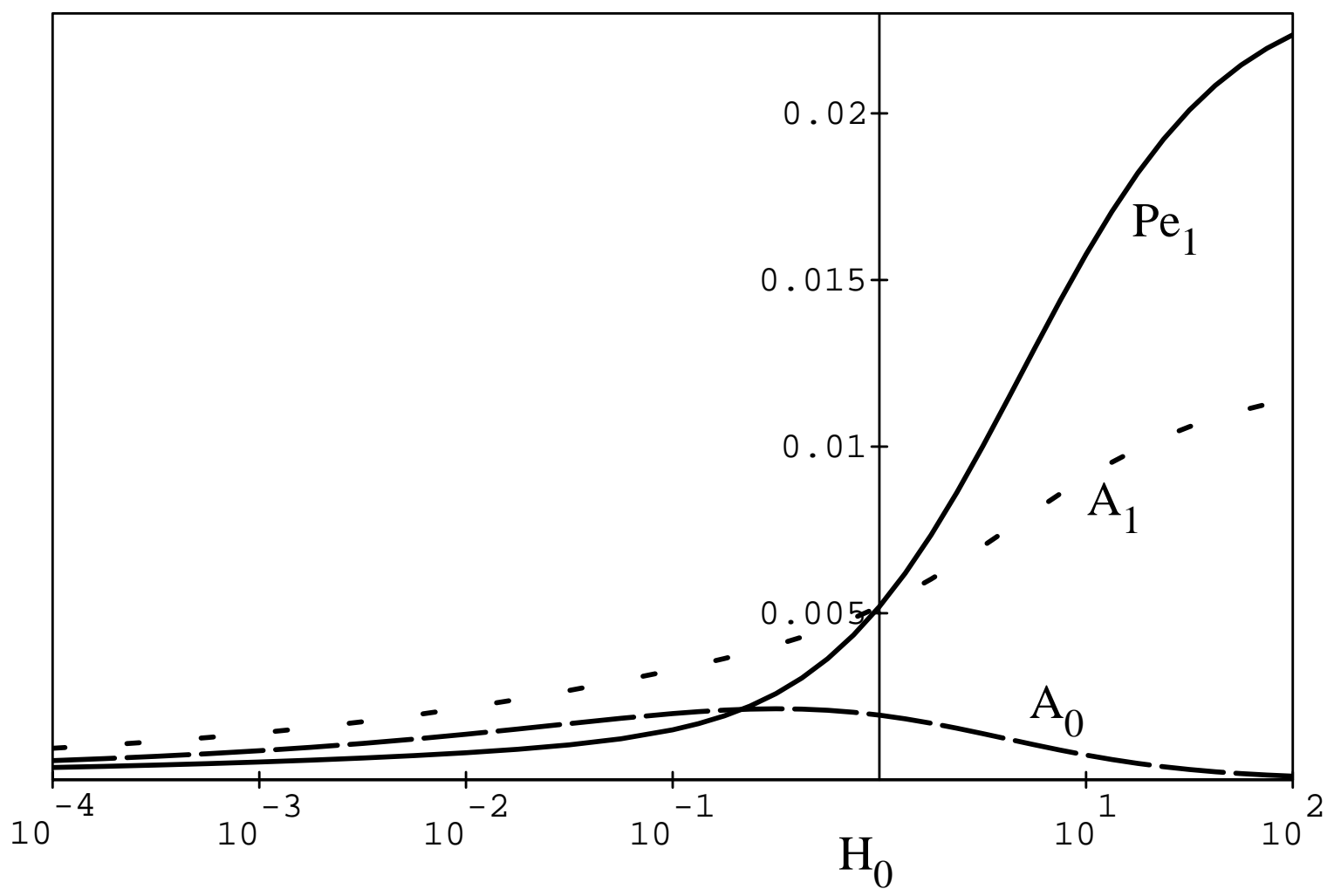

Figure 5: Interface perturbations $A_{0}$ (long dashes) and $A_{1}$ (short dashes) and the relative Peclet number perturbation $P e_{1}=A_{1}-\left(1-H_{0}\right) A_{0}$ (solid) as functions of $H_{0}$ for different Prandtl numbers $P$ : (a) $P=20$. For $P=0.02$, there is a range where $P e_{1}<0$. 


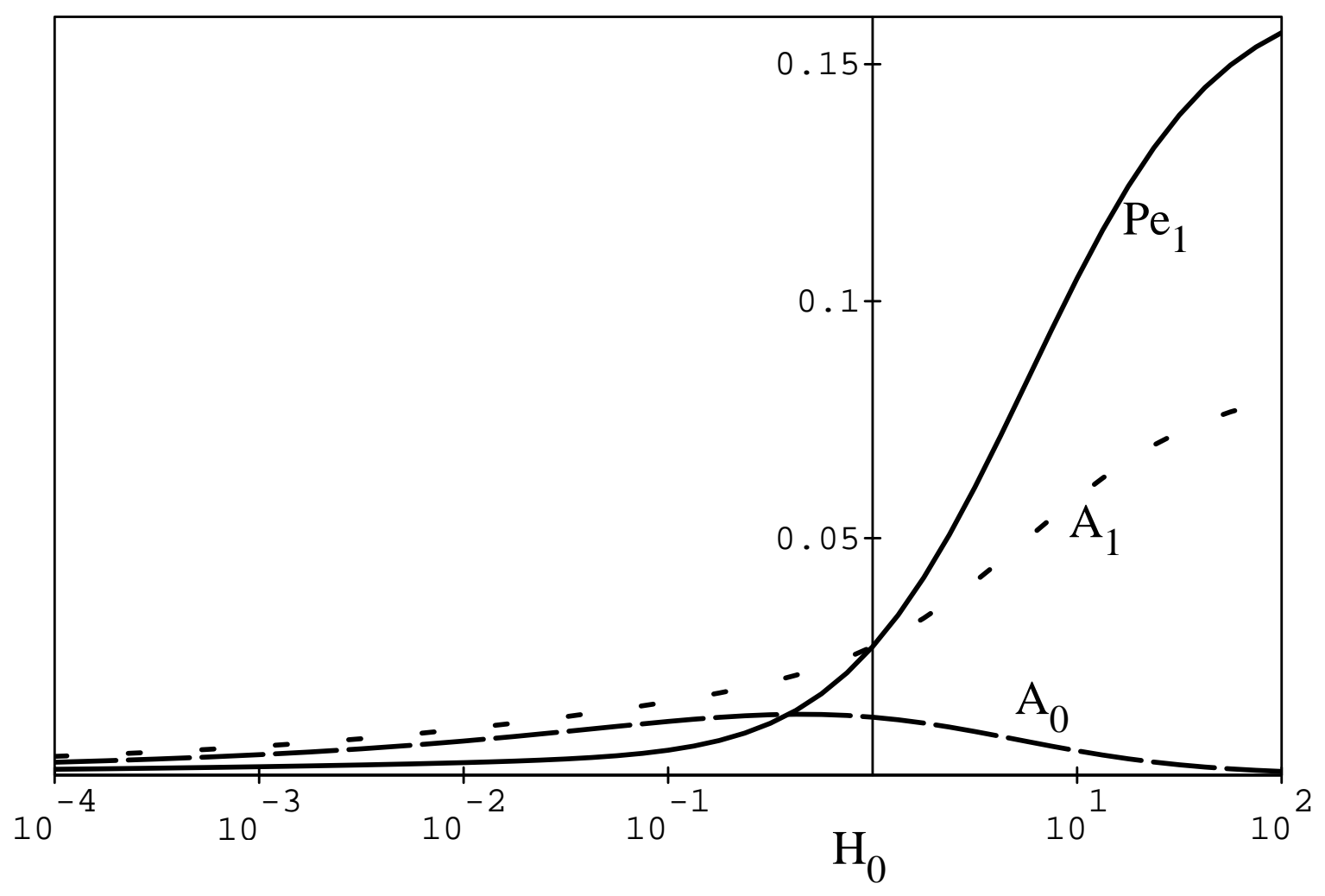

Figure 5: (b) $P=2$. 


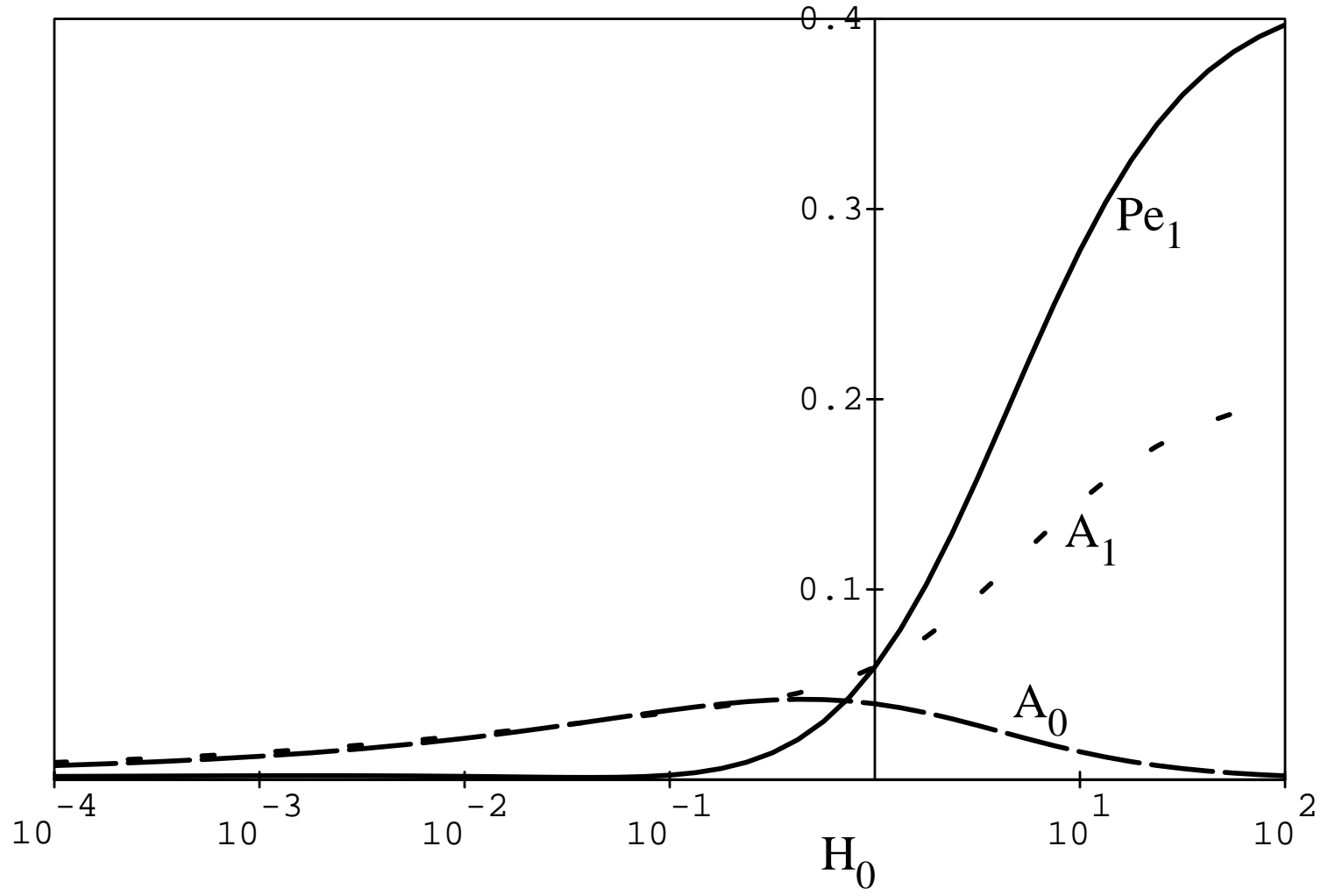

Figure 5: (c) $P=0.2$. 


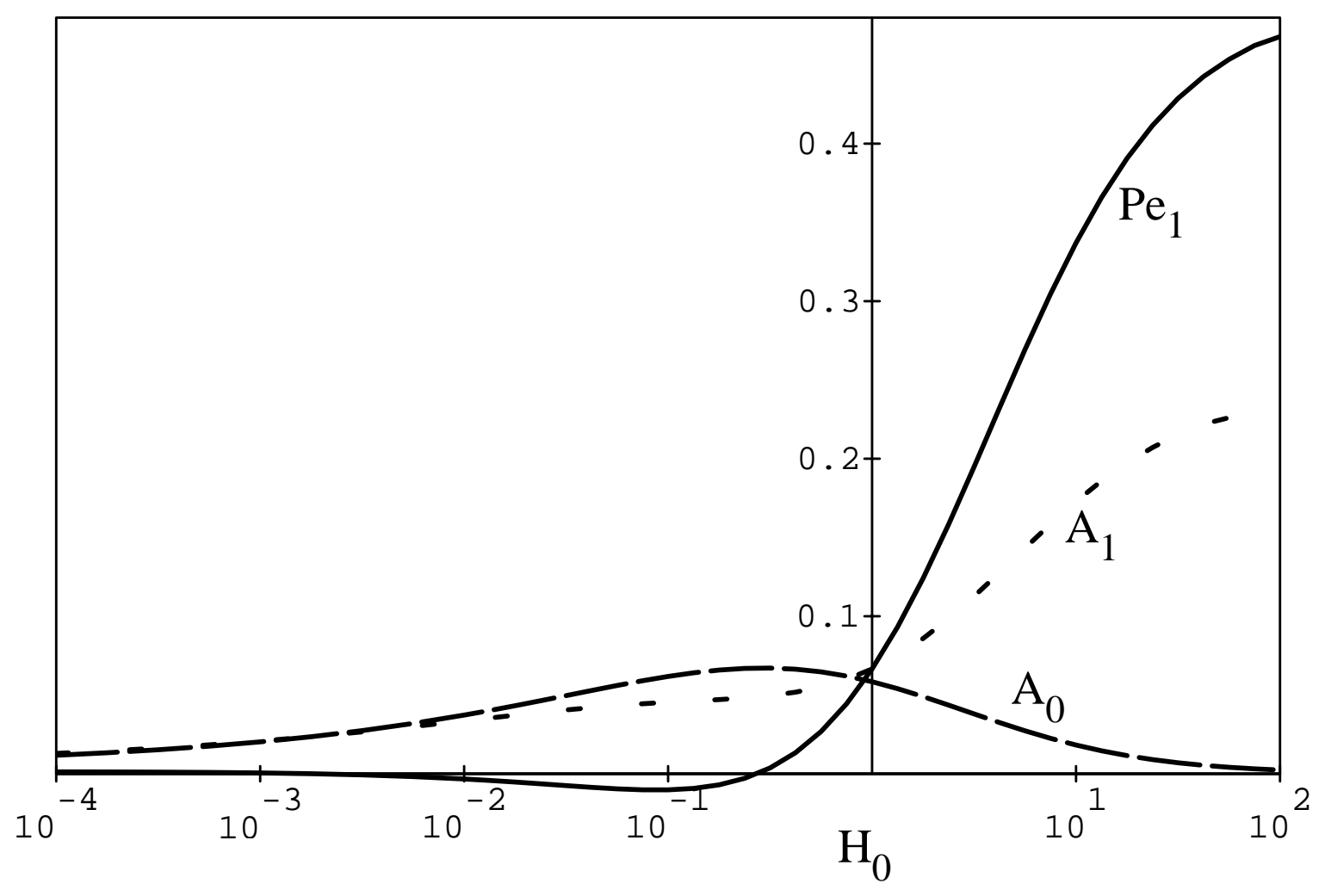

Figure 5: (d) $P=0.02$. 


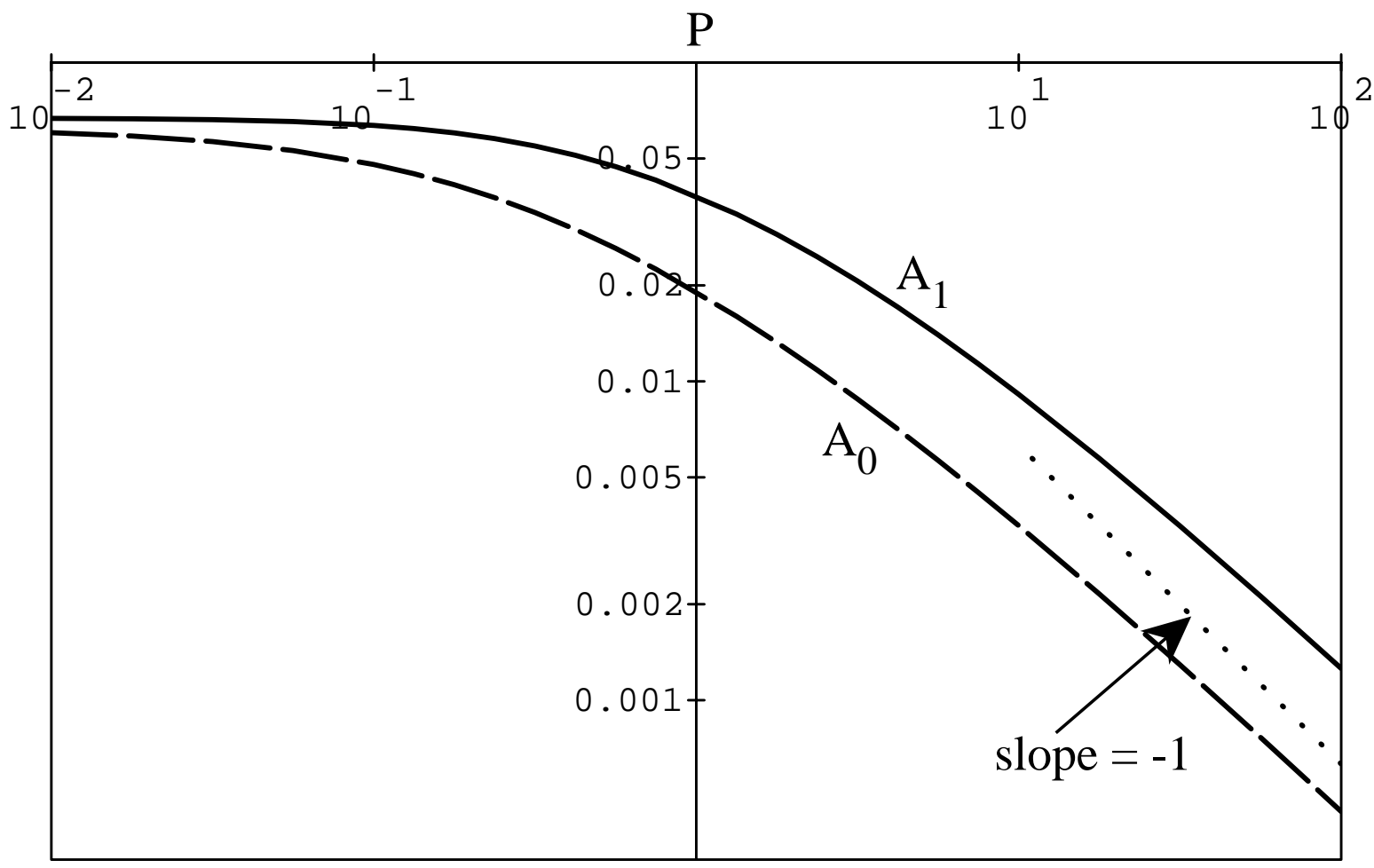

Figure 6: Interface perturbations $A_{0}$ (solid) and $A_{1}$ (dashed) as functions of $P$, for $H_{0}=1$. (a) For large $P$ (at constant $G$ ) the perturbations appear to decrease like $P^{-1}$. This is due to the choice of scaling, as discussed in the text. 


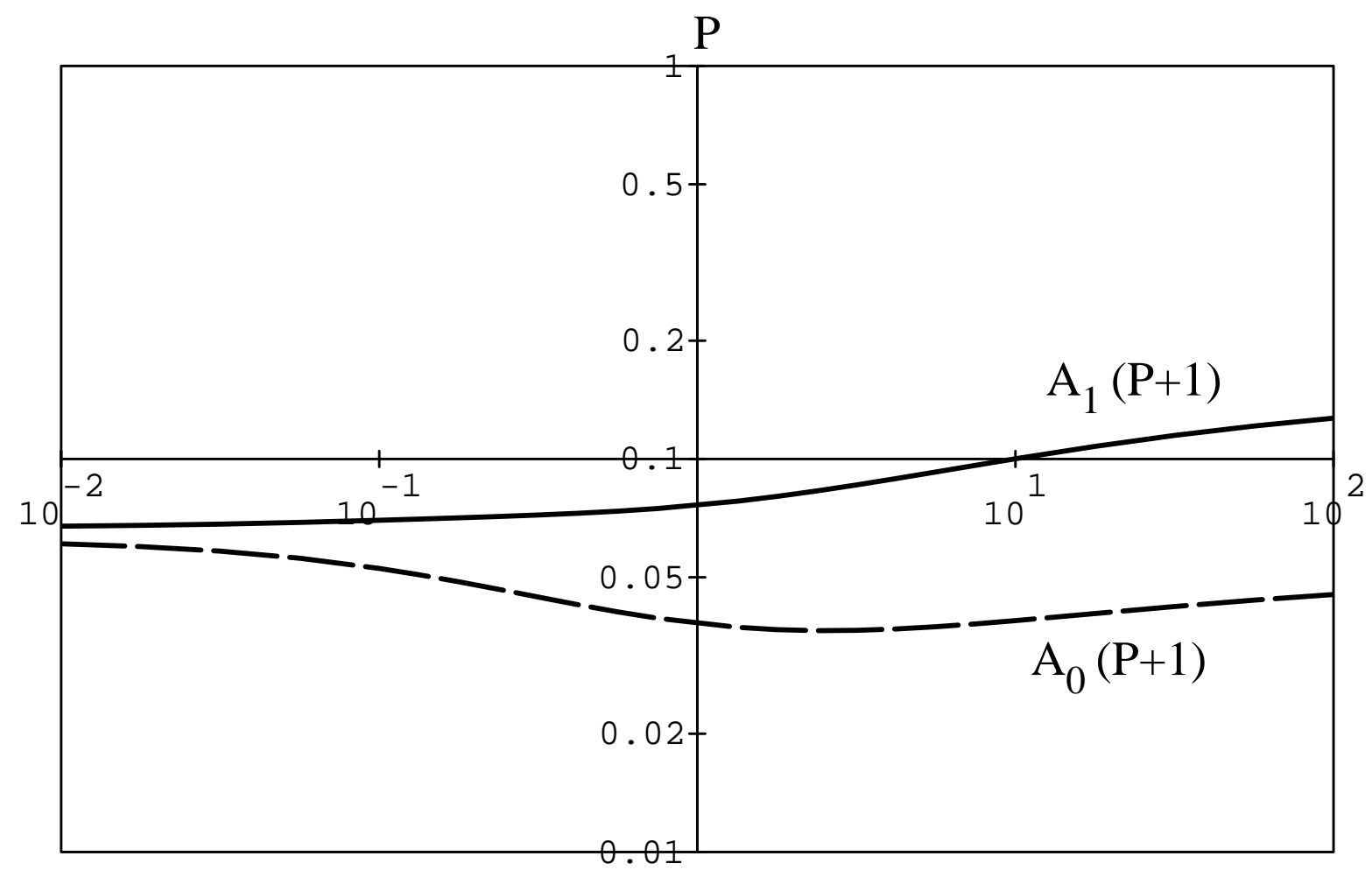

Figure 6: (b) Rescaling by $(P+1)$ corresponds more closely to holding the strength of buoyancy constant. Then the effect, relative to this rescaling, of increasing $P$ is to increase $A_{1}$ while decreasing $A_{0}$. 

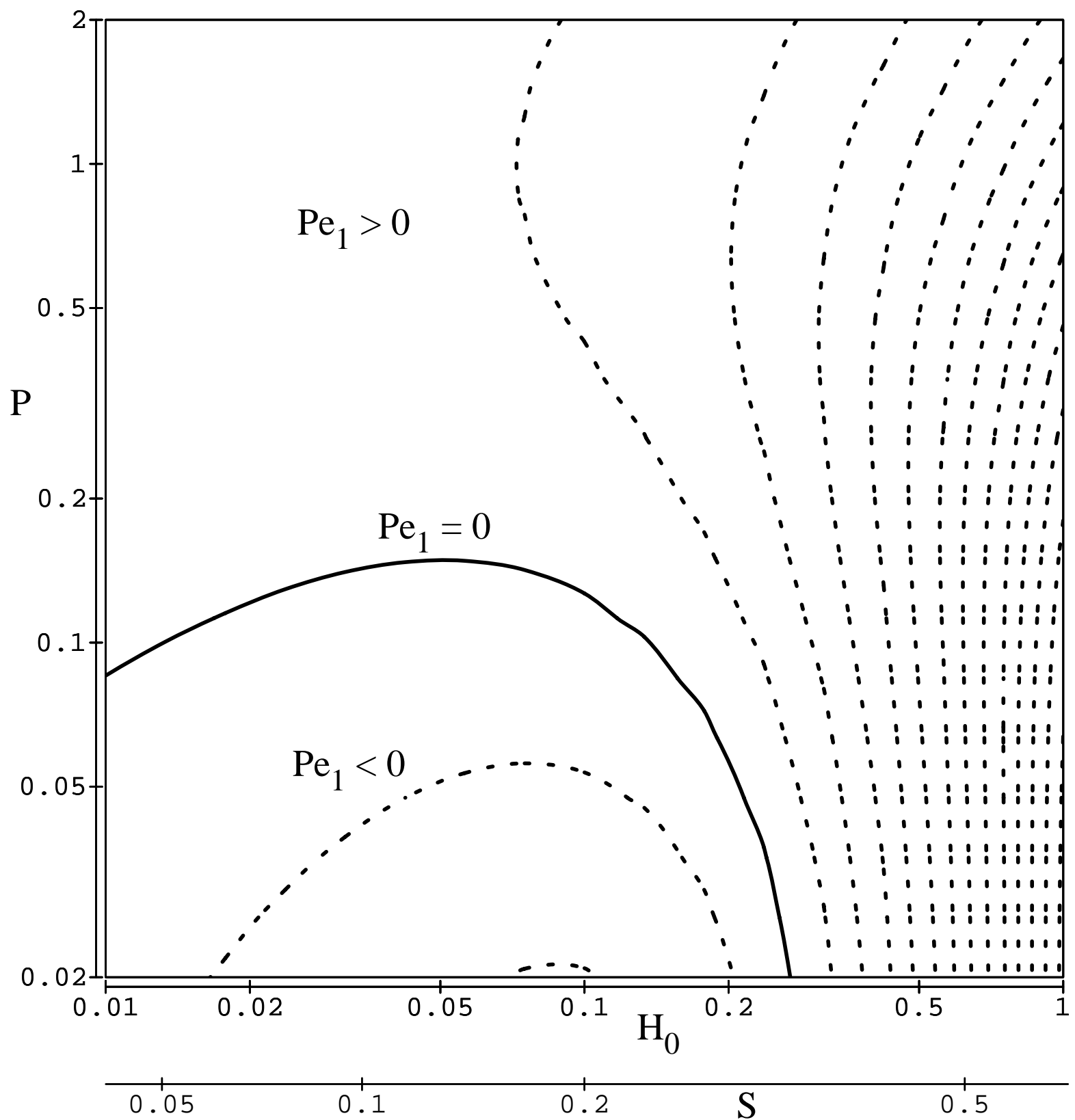

Figure 7: Sign of perturbation: In the $H_{0}, P$ parameter plane the curve $P e_{1}=0$ separates (to $\mathrm{O}(G)$ ) the region where buoyancy enhances tip growth $\left(P e_{1}>0\right)$ from the other region where buoyancy reduces tip growth $\left(P e_{1}<0\right)$. Also shown are other contours (dashed) of constant $P e_{1}$ at equal intervals of 0.005 . Note that the scale for $S$, shown for comparison, is not a regular logarithmic scale. 


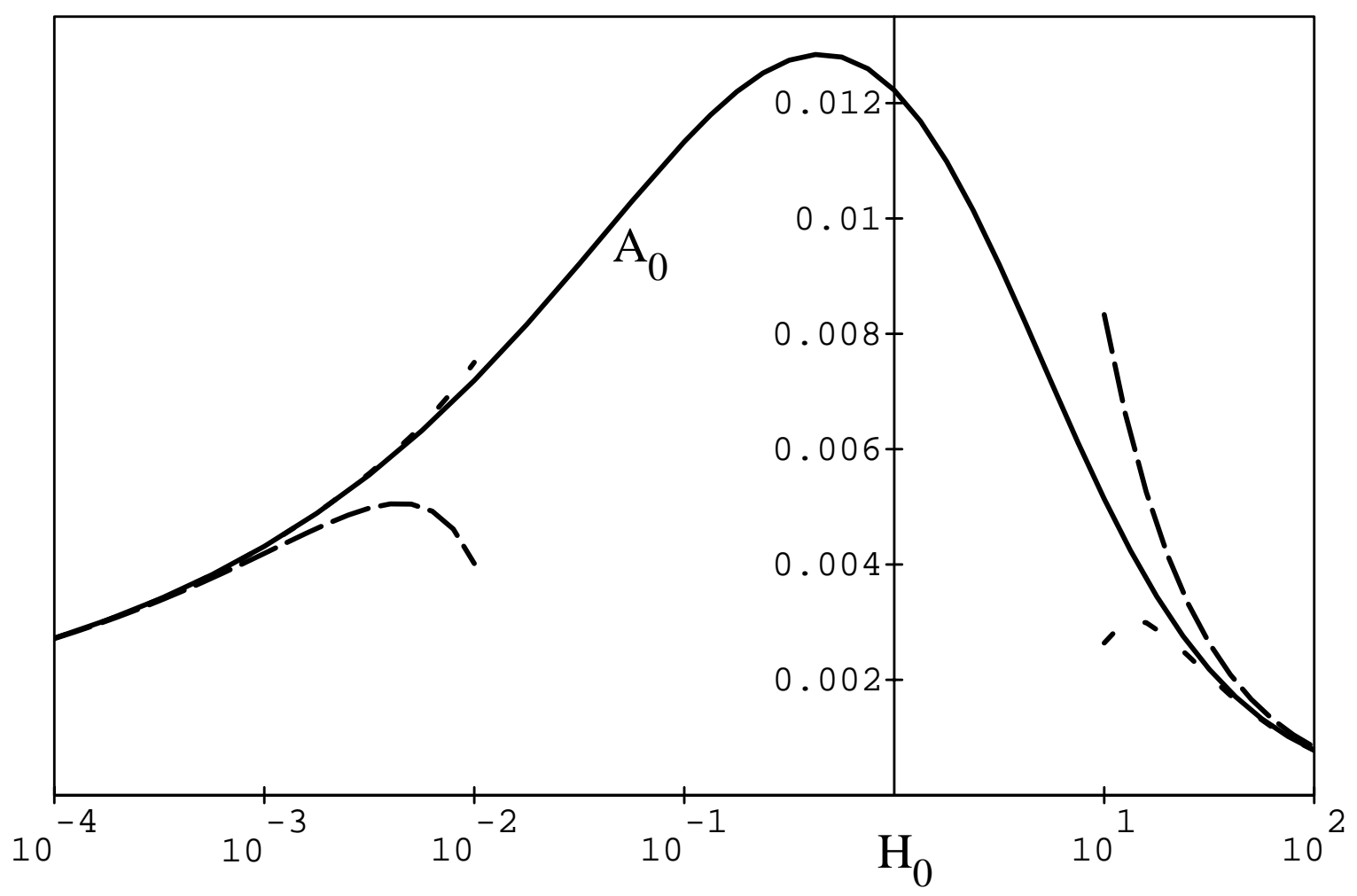

Figure 8: Asymptotic approximations: For $P=2, A_{0}(8 \mathrm{a})$ and $A_{1}(8 \mathrm{~b})$ are shown (solid), with small- $H_{0}$ asymptotic forms to $\mathrm{O}(1)$ (as in Eq. 56) (long dashes) and to $\mathrm{O}\left(H_{0}\right)$ (short dashes), and large- $H_{0}$ asymptotic forms using one term (long dashes) and two terms (see Eq. 55) (short dashes). (a) $A_{0}$. 


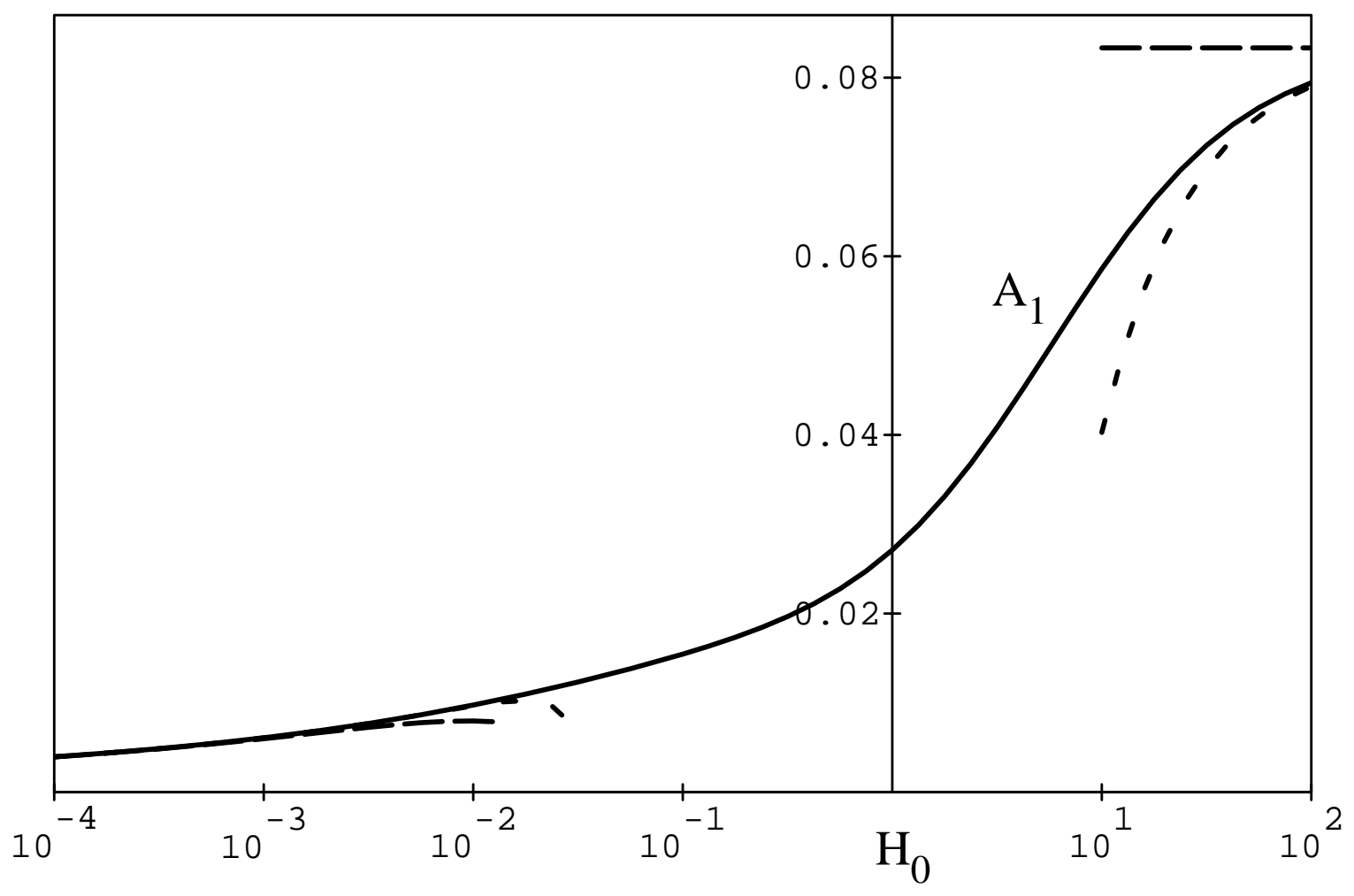

Figure 8: (b) $A_{1}$. 


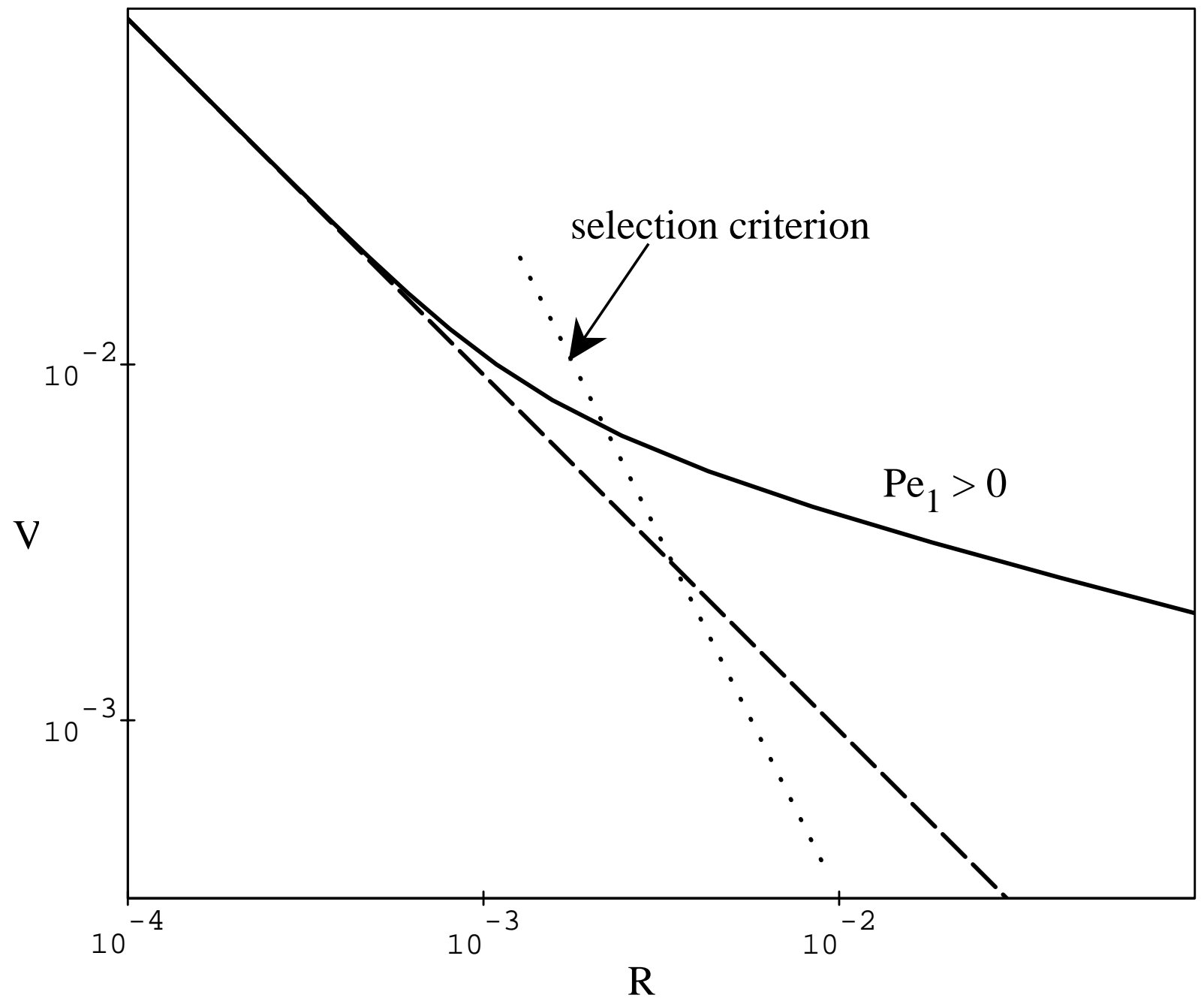

Figure 9: Comparison with Ivantsov solution: The relation between growth speed $V(\mathrm{~cm} / \mathrm{s})$ and tip radius $R$ $(\mathrm{cm})$ for one dimensionless perturbation solution (varying $G$ ) is shown: perturbation theory (solid), Ivantsov solution (dashed), and the selection criterion $\sigma^{*}=0.0195$ (dotted). (a) $S=0.02, H_{0}=0.004, P=22.8$, using material constants for succinonitrile; in this case buoyancy enhances growth. 


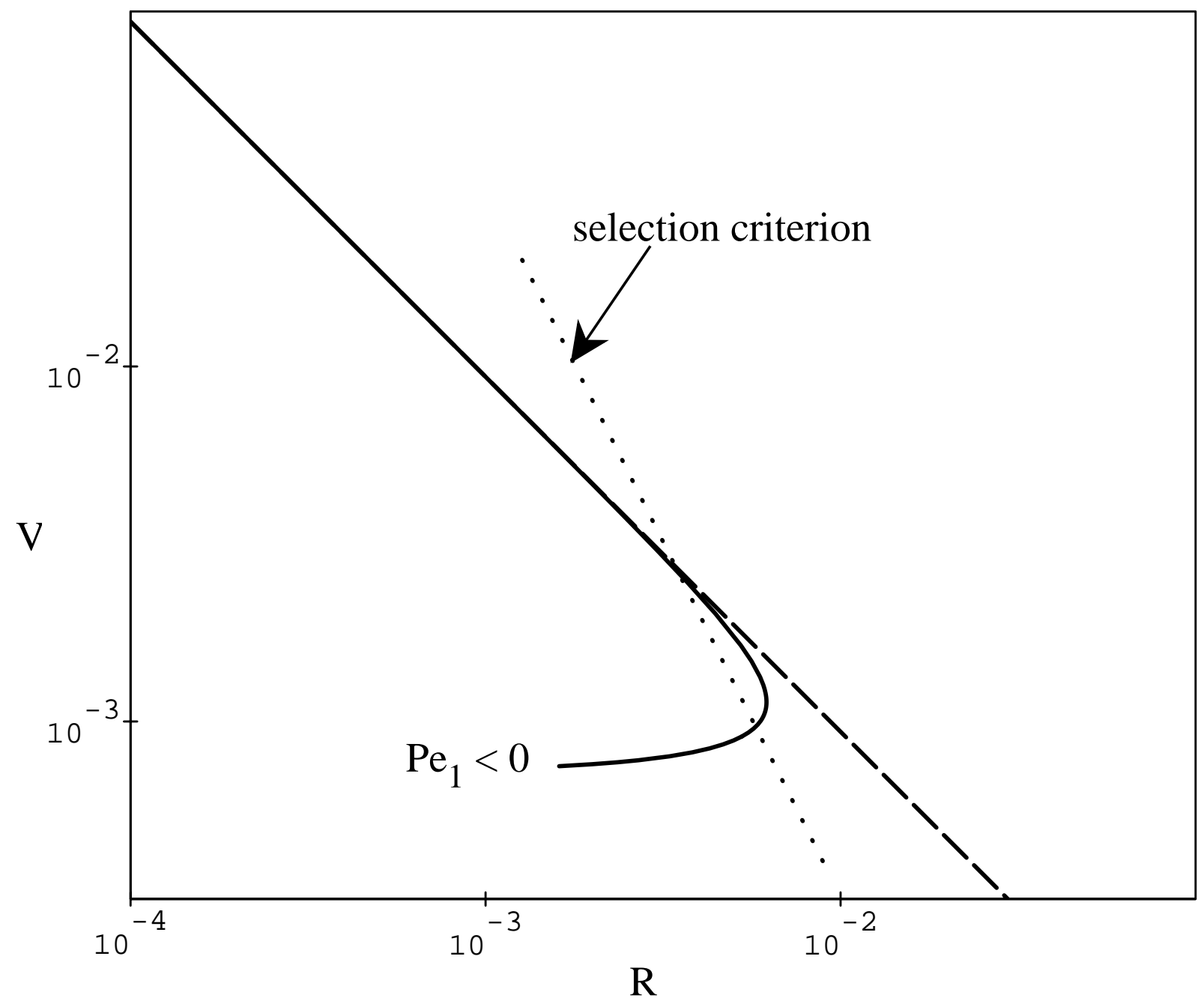

Figure 9: (b) same as (a) except $P=0.0441$, and growth is slightly diminished. 


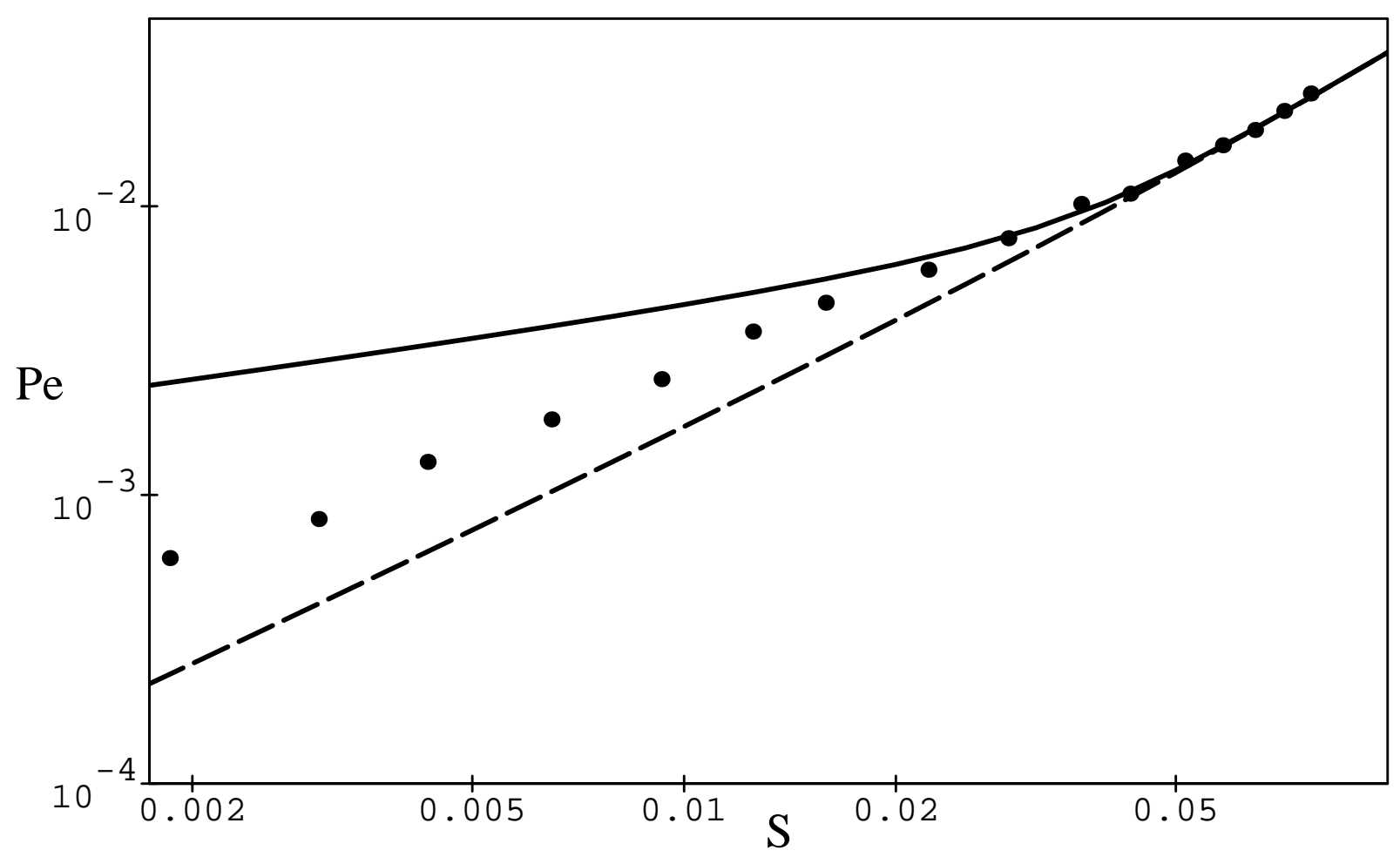

Figure 10: Comparison with experiments: The perturbation theory (solid) and the Ivantsov solution (dashed) (both using the selection criterion $\sigma^{*}=0.0195$ ) are shown with experimental data points (dots) from Ref. [2]. In each figure the perturbation theory departs from the experimental data near the experimental point at $S=0.022$, for which $G=1302$. (a) Peclet number $P e$ versus Stefan number $S$. 


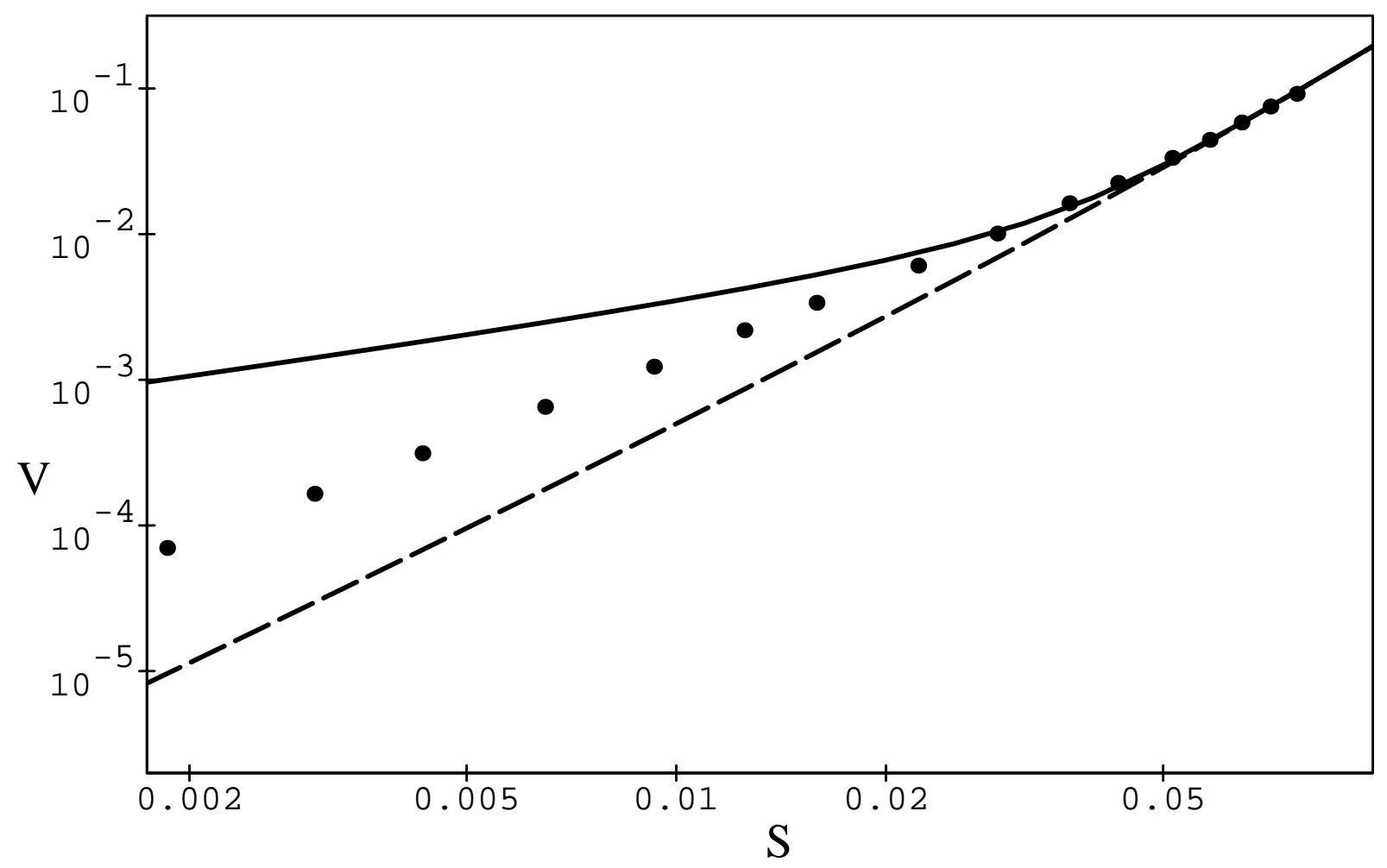

Figure 10: (b) growth speed $V(\mathrm{~cm} / \mathrm{s})$ versus Stefan number $S$. 


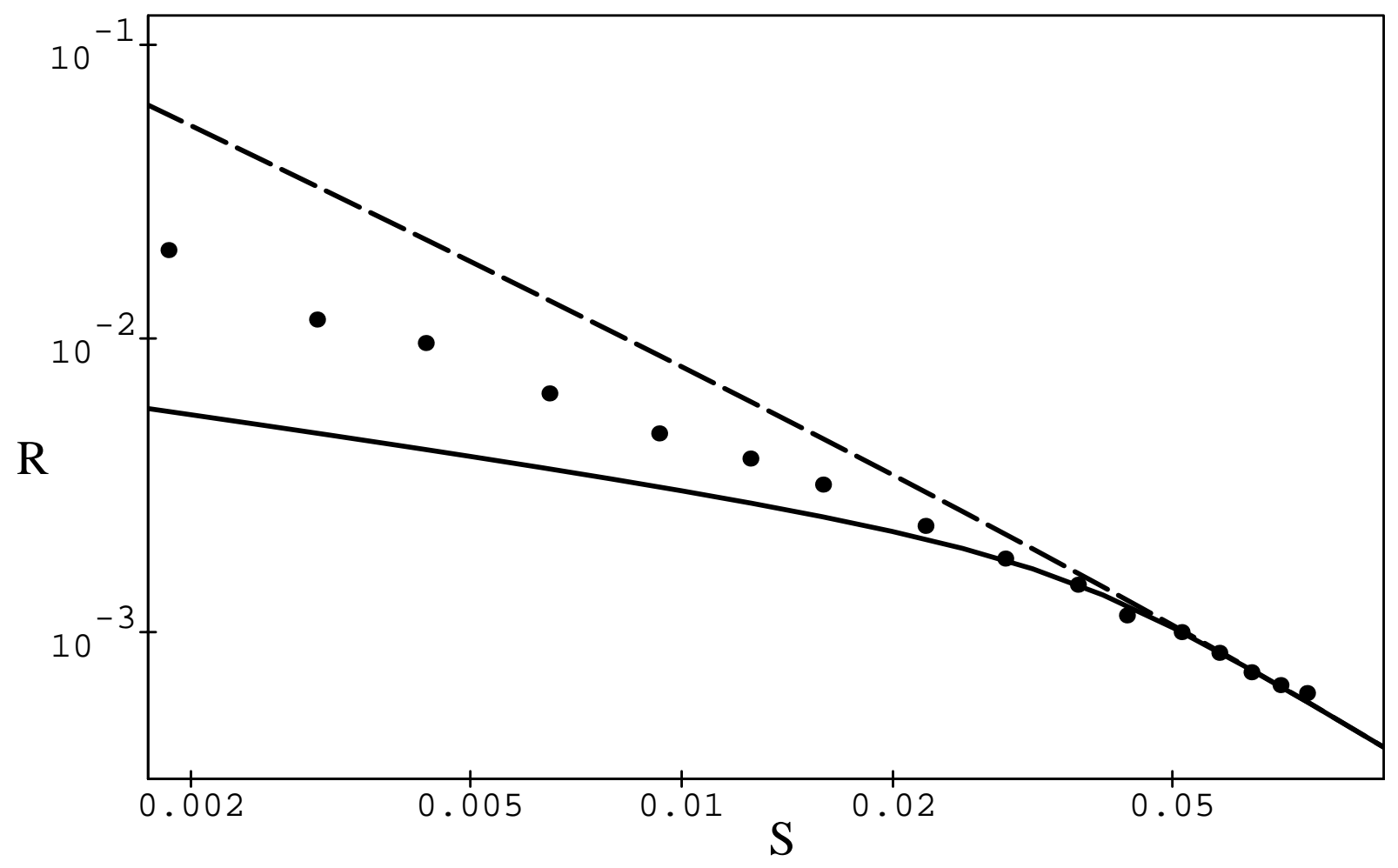

Figure 10: (c) tip radius $R(\mathrm{~cm})$ versus Stefan number $S$.

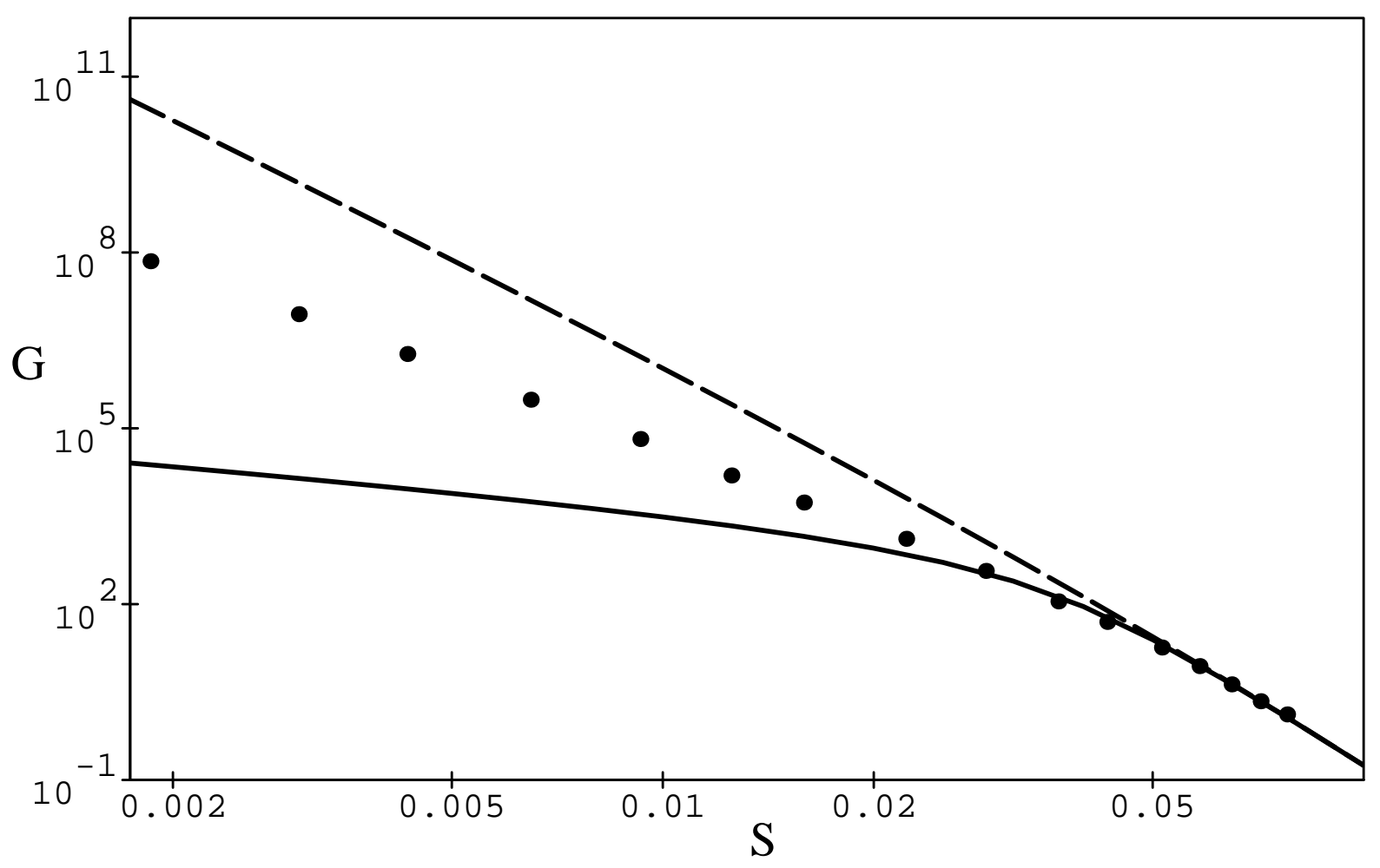

Figure 10: (d) buoyancy parameter $G$ versus Stefan number $S$. 


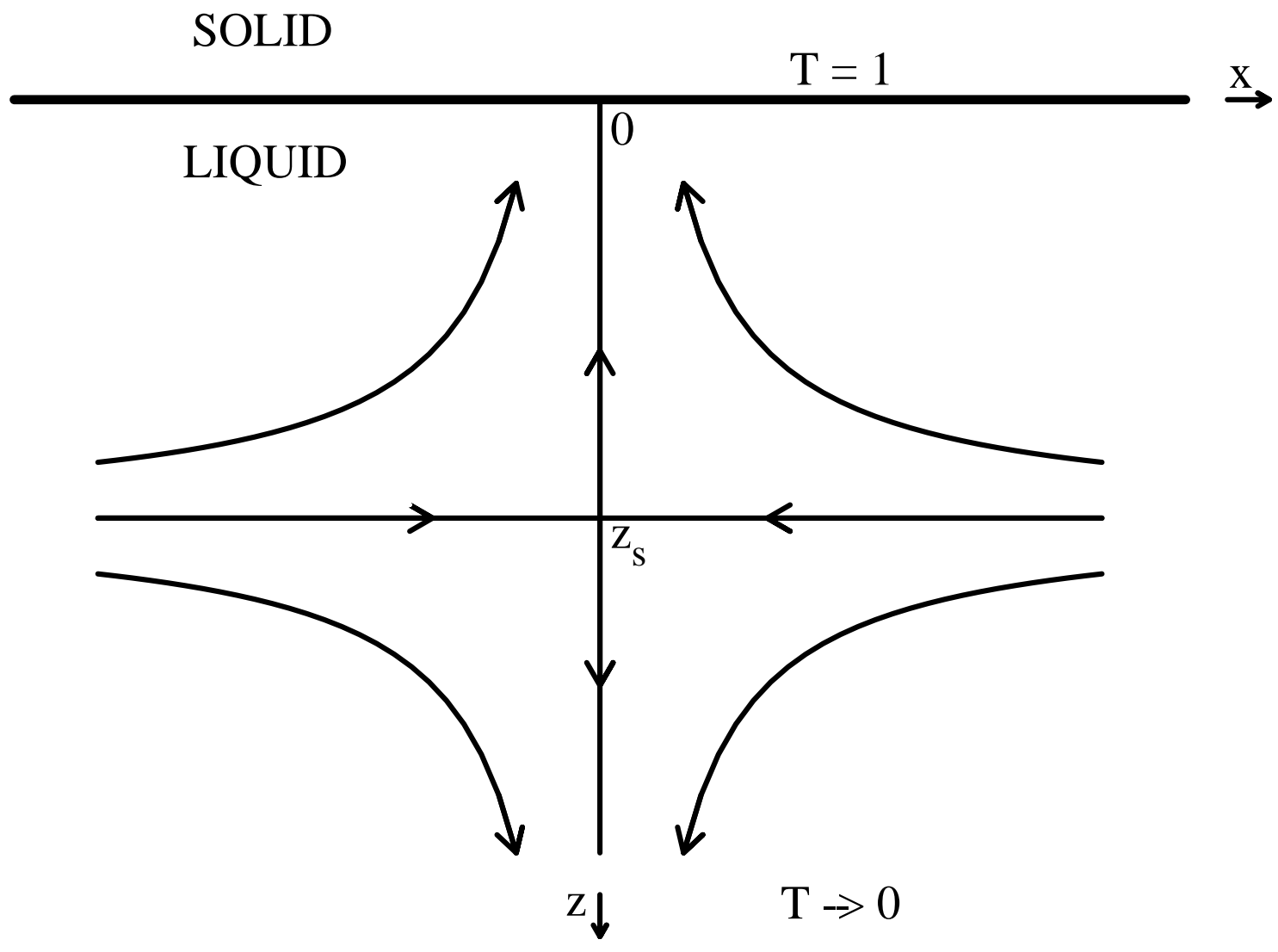

Figure 11: Planar example: As liquid solidifies, the planar interface moves steadily downward with unit speed; the axes $(x, z)$ move with the interface. A weak stagnation-point flow, centered at $\left(0, z_{s}\right)$, is imposed. 